\title{
Stability in a two-dimensional combustion model ${ }^{\dagger}$
}

\author{
JOSEPHUS HULSHOF ${ }^{*}$ \\ Faculty of Sciences, Mathematics and Computer Sciences division, \\ Vrije Universiteit, De Boelelaan 1081, 1081 HV Amsterdam, The Netherlands
}

AND

LUCA LORENZI ${ }^{\S}$

Dipartimento di Matematica, Università di Parma, Via M. D'Azeglio 85/A, 43100 Parma, Italy

[Received 5 February 2003 and in revised form 28 October 2003]

\begin{abstract}
We study the stability of a planar travelling wave in the two-dimensional NEF-combustion model when the reduced Lewis number is equal to zero. The functional analytic setting consists of spaces of suitably exponentially weighted Hölder continuous functions. By exploiting the appearance of the integrated Burgers equation in the equations for perturbations of the wave we avoid the usual assumption that the perturbation must be localized in the lateral space coordinate.
\end{abstract}

Keywords: Free boundary problems; stability of travelling waves; fully nonlinear parabolic problems.

2000 Mathematics Subject Classification: 35K05, 35R35, 35K60, 35B65, 35B35, 37C75, 80A25.

\section{Introduction}

In the 1980s Sivashinsky introduced and studied a two-dimensional thermo-diffusive model for flames in gaseous mixtures. This model is based on the assumption that the diffusion coefficients of the gas and the temperature are almost equal (Nearly Equidiffusional Flame theory), and on the assumption that the normalized activation energy $\mathcal{Z}$ is very large. With the combustion confined to a thin zone of order $\mathcal{Z}^{-1}$, this leads to the following free boundary problem for the reduced temperature $\Theta$, the reduced enthalpy $S$, and the front $\phi$ :

$$
\begin{cases}\frac{\partial \Theta}{\partial t}=\Delta \Theta, & t>0, y \in \mathbb{R}, \eta<\phi(t, y), \\ \Theta=1, & t>0, y \in \mathbb{R}, \eta \geqslant \phi(t, y), \\ \frac{\partial S}{\partial t}=\Delta S-\lambda \Delta \Theta, & t>0, y \in \mathbb{R}, \eta \neq \phi(t, y), \\ {[\Theta]=[S]=0,} & t>0, y \in \mathbb{R}, \\ {\left[\frac{\partial \Theta}{\partial n}\right]=-\exp (S), \quad\left[\frac{\partial S}{\partial n}\right]=\lambda\left[\frac{\partial \Theta}{\partial n}\right],} & t>0, y \in \mathbb{R},\end{cases}
$$

\footnotetext{
$\dagger$ Work partially supported by the research project "Analysis and control of deterministic and stochastic evolution equations" of the Ministero dell'Istruzione, dell'Università e della Ricerca (M.I.U.R.) and by the research project: "Research Training Network (RTN): Fronts-Singularities (RTN contract: HPRN-CT-2002-00274)".

Email: jhulshof@cs.vu.nl

${ }^{\S}$ Email: luca.lorenzi@unipr.it
} 
where $n$ denotes the outward normal to the surface $\eta=\phi(t, y)$. The differential equations for $\Theta$ and $S$ as well as the boundary conditions are coupled by a real parameter $\lambda$ which is a dimensionless constant proportional to the reduced Lewis number. The variables $\Theta$ and $S$ arise as the first relevant terms in the NEF approach for the thermo-diffusive model for temperature $T$ and mass fraction $Y$. This system consists of the reaction-diffusion equations

$$
\frac{\partial T}{\partial t}=\Delta T+\omega(Y, T), \quad \frac{\partial Y}{\partial t}=\frac{1}{\mathrm{Le}} \Delta Y-\omega(Y, T), \quad \omega(Y, T)=Y \exp \left(-\frac{\mathcal{Z}}{T}\right) .
$$

With the enthalpy defined as $H=T+Y$, this approach is based on expanding $T$ and $H$ as formal power series in the reciprocal of $\mathcal{Z}$, coupling the Lewis number Le to $\mathcal{Z}$ as $\mathrm{Le}^{-1}=1-\mathcal{Z}^{-1}$ le, where le is the reduced Lewis number. In this expansion $H_{0}$ is a constant (given by the limits of $T$ and $Y$ in front of the flame front). The NEF model is then derived for $T_{0}$ and $H_{1}$. Here we conform with the notation in [3], where $\Theta=T_{0}, H_{1}=2 S$ and the reduced Lewis number le is replaced by $-2 \lambda$. We refer the reader to the original paper by Sivashinsky [11] for more details on the physical aspects of this model and to [3] for a more mathematical discussion of (1.1).

For any $\lambda \in \mathbb{R}$ problem (1.1) admits a unique (up to translations) planar (i.e. independent of $y$ ) travelling wave (TW) solution given by $(t, \eta) \mapsto\left(\phi(t), \Theta_{\lambda}^{0}(\eta+t), S_{\lambda}^{0}(\eta+t)\right)$, with

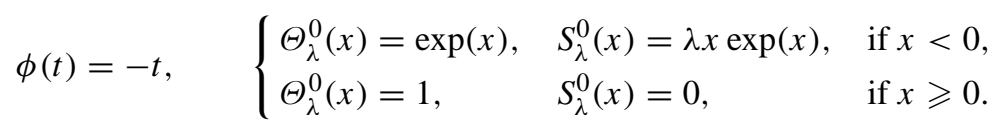

Stability/instability of this solution under two-dimensional perturbations is of physical relevance. The formal study, made by Sivashinsky in [11], showed that there exists a critical value $\lambda_{c}<0$ of $\lambda$ such that the planar TW should be orbitally stable for $\lambda \in\left(\lambda_{c}, 1\right)$ and orbitally unstable for $\lambda<\lambda_{c}$ and for $\lambda>1$.

The instability of the TW was proved in [3] with respect to small and sufficiently smooth perturbations in the context of Hölder spaces. However, the role of the Kuramoto-Sivashinsky equation, derived in this context by Sivashinsky for the description of cellular instabilities for $\lambda \geqslant 1$, seems to remain out of reach of rigorous mathematical methods. We note that whereas in the unstable case the Kuramoto-Sivashinsky equation is expected to play a role, in the stable case this role is played by the integrated Burgers equation. In the context of bistable reaction-diffusion systems both cases are discussed on a completely formal and heuristic level in [6].

Next, in [2] the first author, in a joint paper, proved stability of the TW in the case where $\lambda=0$, assuming to perturb only the temperature $\Theta$. The quadratic term in the integrated Burgers equation appearing in the leading order terms in the perturbation analysis is absorbed in the linear terms by means of a Cole-Hopf bifurcation. Remarkably this makes the usual assumption that perturbations are localized in the lateral $y$-direction redundant, which may be interpreted as an a posteriori validation of the role of the integrated Burgers equation.

More recently, in [8,9] the second author, in a joint paper, showed stability of the TW for $\lambda \in$ $\left(\lambda_{c}, 1\right)$ in the context of weighted Hölder spaces in a slightly different model where the nonlinear term $\exp (S)$ is replaced by $f(S)=1+S+O\left(S^{k}\right)$ for some $k>5$. They assume that the weight function depends both on $\eta$ and $y$ and do not use any explicit form of (part of) the quadratic terms. Here the analogy (of the $\lambda=0$ case) with [5] in the (diagonal) reaction-diffusion context should be noted, where the localization assumption appears in a different form (in view of the use of Sobolev 
spaces). We emphasize that such results do not fully generalize the one-dimensional results which allow perturbations converging to a translate of the wave.

Here we generalize the result of [2] to the NEF model with $\lambda=0$, allowing perturbations on $\Theta$ and $S$ as well. We prove that the TW solution is stable with respect to suitable weighted (in $x$ only) smooth perturbations.

To prove our stability results, we transform the problem (by suitable changes of coordinates and unknowns) into an equivalent one of the form

$$
\begin{cases}D_{t} \mathbf{u}(t, x, y)=\mathcal{L} \mathbf{u}(t, x, y)+\mathcal{F}(\mathbf{u}(t, \cdot))(x, y), & t \geqslant 0,(x, y) \in \mathbb{R}_{-}^{2}, \\ \mathcal{B u}(t, \cdot)(y)=\mathcal{G}(\mathbf{u}(t, \cdot))(y), & t \geqslant 0, y \in \mathbb{R}\end{cases}
$$

set in the fixed space domain $\mathbb{R}_{-}^{2}:=\mathbb{R}_{-} \times \mathbb{R}$, where $\mathcal{L}$ is a second order operator and $\mathcal{B}$ is a first order boundary operator, both with constant coefficients. The nonlinear and nonlocal operators $\mathcal{F}$ and $\mathcal{G}$ are quadratic near 0 . Now, the problem of the stability of the TW for problem (1.1) is transformed into the problem of the stability of the null solution to problem $(1.3)$.

The realization $L$ of the operator $\mathcal{L}$ in the space of all bounded and continuous functions generates an analytic semigroup and its spectrum consists of all the $\omega \in \mathbb{C}$ such that $\operatorname{Re} \omega \leqslant$ $-(\operatorname{Im} \omega)^{2}$. Hence the spectrum of $L$ is contained in the left half-plane and 0 is an accumulation point of eigenvalues. Hence we are in a critical case of stability and we cannot invoke the linearized stability principle to prove our stability results.

Working with a space $X$ of weighted continuous functions (corresponding in our situation to an exponential weight function in the $x$-variable) allows us to limit the continuous spectrum to the half-line $(-\infty, 0]$, but hereby we remain in the critical case of stability. The key idea to overcome this difficulty consists in determining a suitable projection, which is not a spectral projection, but enjoys most of the typical properties of a spectral projection, namely we determine a projection $P$ such that

$$
\left\|D_{t}^{\alpha_{1}} D_{x}^{\alpha_{2}} D_{y}^{\alpha_{3}}(I-P) e^{t L}\right\|_{L(X)} \leqslant M e^{-\omega t}, \quad \forall t \geqslant 0
$$

for some positive constant $\omega$ and for any multindex $\alpha=\left(\alpha_{1}, \alpha_{2}, \alpha_{3}\right)$, where by $e^{t L}$ we denote the (analytic) semigroup generated by the operator $L$. To construct the projection $P$ we observe that the operator $L$ can be split into the sum of two operators $L_{1}$ and $L_{2}$ acting, respectively, on the $x$ and the $y$-variable, which commute in the resolvent sense. Since 0 is a simple eigenvalue of $L_{1}$, we can find the spectral projection $P$ corresponding to the eigenvalue 0 of $L_{1}$. Such a projection is readily seen to satisfy (1.4). To carry out our construction of the projection $P$, it is essential that $L$ can be split into the sum of two operators commuting in the resolvent sense. As pointed out in the appendix, this is the case only if $\lambda=0$, where the system of differential equations in (1.1) and the operator $\mathcal{L}$ in 1.3 are in diagonal form.

Splitting the solution $\mathbf{u}$ to the initial value problem for 1.3$)$ along $P(X)$ and $(I-P)(X)$ we can write $\mathbf{u}(t, x, y)=r(t, y)\left(e^{x}, 0,0\right)+\mathbf{w}(t, x, y)$. This position allows us to decouple problem 1.3 into two new problems for the pair $(r, \mathbf{w})$. The differential equation in the first system is a nonlinear Burgers equation. The second system is set in the stable manifold $(I-P)(X)$ where the semigroup satisfies (1.4). What we expect is that $\mathbf{w}$ and its derivatives decay at least with polynomial rate at infinity, and this is just the case. Hence the asymptotic behaviour of the solution to problem (1.1) is determined by the behaviour near infinity of the solution to the Cauchy problem associated with the nonlinear Burgers equation. Such an equation contains the term $r_{y}^{2}$ which is critical for the 
stability. Performing a suitable Cole-Hopf transformation allows us to eliminate this term. We get a new equation for the new unknown $q$, namely a nonlinear heat equation whose solution exhibits the same decay estimates at infinity as the linear heat equation does. Hence $q$ stays bounded in $\mathbb{R}_{+} \times \mathbb{R}$ while its derivatives decay polynomially when $t$ approaches infinity.

Coming back to our original problem (1.1) we can conclude that the solution $(\Theta, S, \phi)$, corresponding to initial data close to the TW, exists globally in time and, in the coordinate system attached to the front, $\Theta, S$ and $\phi$ stay bounded and sufficiently close to the TW, while their space and time derivatives decay with polynomial rate at infinity.

\section{Reduction to a fixed boundary problem}

In this section we transform our problem into an equivalent one of the type (1.3). First of all we fix the boundary by setting $\Theta^{1}(t, x, y)=\Theta(t, x+\phi(t, y), y), S^{1}(t, x, y)=S(t, x+\phi(t, y), y)$. Moreover, we set $\varphi(t, y)=\phi(t, y)+t$. Easy computations show that the triplet $\left(\Theta^{1}, S^{1}, \varphi\right)$ solves the following problem:

$$
\begin{gathered}
\Theta_{t}^{1}+\Theta_{x}^{1}=\Delta \Theta^{1}+\left(\varphi_{y}\right)^{2} \Theta_{x x}^{1}-2 \varphi_{y} \Theta_{x y}^{1}+\left(\varphi_{t}-\varphi_{y y}\right) \Theta_{x}^{1}, \quad x<0, \\
\Theta^{1}=1, \quad x \geqslant 0, \\
S_{t}^{1}+S_{x}^{1}=\Delta S^{1}+\left(\varphi_{y}\right)^{2} S_{x x}^{1}+\left(\varphi_{t}-\varphi_{y y}\right) S_{x}^{1}-2 \varphi_{y} S_{x y}^{1}, \quad x \neq 0, \\
{\left[\Theta^{1}\right]=\left[S^{1}\right]=\left[S_{x}^{1}\right]=0, \quad\left[\Theta_{x}^{1}\right]=-\left(1+\varphi_{y}^{2}\right)^{-1 / 2} e^{S^{1}} .}
\end{gathered}
$$

Here [ $\cdot$ ] denotes the jump at $x=0$, and $\Delta=D_{x}^{2}+D_{y}^{2}$. To decouple the system we argue as in [3] introducing the new unknowns $v$ and $w$ defined by

$$
\begin{aligned}
\text { (i) } \Theta^{1}(t, x, y) & =\Theta^{0}(x)+\Theta_{x}^{0}(x) \varphi(t, y)+v(t, x, y) \\
\text { (ii) } \quad S^{1}(t, x, y) & =w(t, x, y)
\end{aligned}
$$

here and throughout the paper we write $\Theta^{0}$ instead of $\Theta_{0}^{0}$ (see $[1.2$ ).

Performing the change of unknowns in 2.5 , we get an equivalent problem for the triplet $(v, w, \varphi)$. But taking the jump of both sides of $(2.5)$ at $x=0$, and recalling that $\left[\Theta^{1}\right]=\left[S^{1}\right]=0$, we get

$$
\varphi(t, y)=-v(t, 0, y), \quad \forall t>0, \forall y \in \mathbb{R}
$$

Setting $\mathbf{u}=(v, w, h)$, where $h(t, x, y)=w(t,-x, y)$ for any $t>0$ and any $(x, y) \in \overline{\mathbb{R}}_{-}^{2}:=$ $\left\{(x, y) \in \mathbb{R}^{2}: x \leqslant 0\right\}$, and replacing the unknown front by its expression in terms of $\mathbf{u}$ given by 2.6, we get the following problem which is equivalent to [2.1]-2.4:

$$
\begin{cases}\mathbf{u}_{t}(t, x, y)=\mathcal{L} \mathbf{u}(t, x, y)+\mathcal{F}_{0}(\mathbf{u}(t, \cdot))(x, y)-v_{t}(t, 0, y) \Psi(\mathbf{u}(t, \cdot))(x, y), & t \geqslant 0,(x, y) \in \overline{\mathbb{R}}_{-}^{2}, \\ (\mathcal{B} \mathbf{u}(t, \cdot))(y)=\mathcal{G}(\mathbf{u}(t, \cdot))(y), & t \geqslant 0, y \in \mathbb{R} .\end{cases}
$$

In 2.7) the second-order differential operator $\mathcal{L}$, the boundary differential operator $\mathcal{B}=$ $\left(B_{0}, B_{1}, B_{2}\right)$ and $\mathcal{F}_{0}(\mathbf{u})=\left(f_{1}(\mathbf{u}), f_{2}(\mathbf{u}), f_{3}(\mathbf{u})\right)$ are given, respectively, by

$$
\begin{aligned}
& \mathcal{L} \mathbf{u}=\left(\Delta v-v_{x}, \Delta w-w_{x}, \Delta h+h_{x}\right) \\
& \left\{\begin{array}{l}
B_{0} \mathbf{u}=w(\cdot, 0, \cdot)-h(\cdot, 0, \cdot) \\
B_{1} \mathbf{u}=w_{x}(\cdot, 0, \cdot)+h_{x}(\cdot, 0, \cdot) \\
B_{2} \mathbf{u}=v(\cdot, 0, \cdot)+h(\cdot, 0, \cdot)-v_{x}(\cdot, 0, \cdot) ;
\end{array}\right.
\end{aligned}
$$




$$
\begin{aligned}
f_{1}(\mathbf{u})= & \left(v_{y}(\cdot, 0, \cdot)\right)^{2}\left(\Theta_{x x}^{0}-v(\cdot, 0, \cdot) \Theta_{x x x}^{0}+v_{x x}\right)+2 v_{y}(\cdot, 0, \cdot)\left(-v_{y}(\cdot, 0, \cdot) \Theta_{x x}^{0}+v_{x y}\right) \\
& +v_{y y}(\cdot, 0, \cdot)\left(-v(\cdot, 0, \cdot) \Theta_{x x}^{0}+v_{x}\right) \\
f_{2}(\mathbf{u})= & \left(v_{y}(\cdot, 0, \cdot)\right)^{2} w_{x x}+2 v_{y}(\cdot, 0, \cdot) w_{x y}+v_{y y}(\cdot, 0, \cdot) w_{x} \\
f_{3}(\mathbf{u})= & \left(v_{y}(\cdot, 0, \cdot)\right)^{2} h_{x x}-2 v_{y}(\cdot, 0, \cdot) h_{x y}-v_{y y}(\cdot, 0, \cdot) h_{x}
\end{aligned}
$$

while $\Psi(\mathbf{u})$ and $\mathcal{G}(\mathbf{u})$ are defined by

$$
\Psi(\mathbf{u})=\left(-v(\cdot, 0, \cdot) \Theta_{x x}^{0}+v_{x}, w_{x},-h_{x}\right)
$$

and

$$
\mathcal{G}(\mathbf{u})=(0,0, g(\mathbf{u})), \quad g(\mathbf{u})=1+h(\cdot, 0, \cdot)-\left(1+\left(v_{y}(\cdot, 0, \cdot)\right)^{2}\right)^{-1 / 2} e^{h(\cdot, 0, \cdot)} .
$$

Problem 2.7) still contains the unknown $v_{t}(t, 0, y)$ in its right-hand side. However, evaluating the first component of the differential equation in (2.7) at $x=0$, we can get $v_{t}(t, 0, y)$ in terms of the space derivatives of $\mathbf{u}$, provided $v_{x}(t, 0, y)-v(t, 0, y) \neq-1$. Thus, we finally get the following problem for $\mathbf{u}$ :

$$
\begin{cases}\mathbf{u}_{t}(t, x, y)=\mathcal{L} \mathbf{u}(t, x, y)+\mathcal{F}(\mathbf{u}(t, \cdot))(x, y), & t \geqslant 0,(x, y) \in \overline{\mathbb{R}}_{-}^{2} \\ (\mathcal{B u}(t, \cdot))(y)=\mathcal{G}(\mathbf{u}(t, \cdot))(y), & t \geqslant 0, y \in \mathbb{R}\end{cases}
$$

where

$$
\mathcal{F}(\mathbf{u}(t, \cdot))=\mathcal{F}_{0}(\mathbf{u}(t, \cdot))-\frac{\Delta v(t, 0, \cdot)-v_{x}(t, 0, \cdot)+f_{1}(\mathbf{u}(t, \cdot))(0, \cdot)}{1-v(t, 0, \cdot)+v_{x}(t, 0, \cdot)} \Psi(\mathbf{u}(t, \cdot)), \quad \forall t>0 .
$$

Note that the TW solution to the original problem corresponds to the null solution to (2.15) and the solutions close to the TW correspond to small solutions to 2.15].

\section{The function spaces}

In this section we introduce the Banach spaces we deal with throughout this paper. For notational convenience we use bold style to denote vector-valued functions. According to the notations of the previous sections, we set $\mathbb{R}_{-}^{2}:=\left\{(x, y) \in \mathbb{R}^{2}: x<0\right\}$ and we denote by $\overline{\mathbb{R}}_{-}^{2}$ its closure in $\mathbb{R}^{2}$. Moreover, for any $\mathbf{f}: \mathbb{R}_{-}^{2} \rightarrow \mathbb{R}^{3}, \mathbf{f}=\left(f, g_{1}, g_{2}\right)$, we denote by $\mathbf{f}^{\sharp}$ the function defined by $\mathbf{f}^{\sharp}(x, y)=\left(e^{-x / 2} f(x, y), e^{-x / 2} g_{1}(x, y), e^{x / 2} g_{2}(x, y)\right)$.

DEFINITION 3.1 For any $k \geqslant 0$ we define the function space $X_{k}$ by

$$
X_{k}=\left\{\mathbf{f}: \overline{\mathbb{R}}_{-}^{2} \rightarrow \mathbb{R}^{3}: \mathbf{f}^{\sharp} \in C_{b}^{k}\left(\overline{\mathbb{R}}_{-}^{2}\right)\right\},
$$

and we endow it with the norm $\|\mathbf{f}\|_{X_{k}}=\left\|\mathbf{f}^{\sharp}\right\|_{C_{b}^{k}\left(\mathbb{R}_{-}^{2}\right)}, \mathbf{f} \in X_{k}$.

Moreover, for any $a, b \in \mathbb{R}_{+} \cup\{+\infty\}, a<b$, and any $\alpha \in(0,1)$ we define the Banach spaces

$$
\begin{aligned}
\mathcal{X}_{\alpha / 2, \alpha}(a, b)= & \left\{\mathbf{u}: \mathbf{u}(t, \cdot) \in X_{\alpha} \forall t \in[a, b], \sup _{a<t<b}\|\mathbf{u}(t, \cdot)\|_{X_{\alpha}}<+\infty,\right. \\
& \left.\mathbf{u}(\cdot, x, y) \in C^{\alpha / 2}([a, b]) \forall(x, y) \in \overline{\mathbb{R}}_{-}^{2}, \sup _{(x, y) \in \mathbb{R}_{-}^{2}}\|\mathbf{u}(\cdot, x, y)\|_{C^{\alpha / 2}([a, b])}<+\infty\right\},
\end{aligned}
$$

$\mathcal{X}_{1+\alpha / 2,2+\alpha}(a, b)=\left\{\mathbf{u}: D_{t}^{\alpha_{1}} D_{x}^{\alpha_{2}} D_{y}^{\alpha_{3}} \mathbf{u} \in \mathcal{X}_{\alpha / 2, \alpha}(a, b)\right.$ for $\left.2 \alpha_{1}+\alpha_{2}+\alpha_{3} \leqslant 2\right\}$. 
They are normed by

$$
\begin{gathered}
\|\mathbf{u}\| \mathcal{X}_{\alpha / 2, \alpha}(a, b)=\sup _{a<t<b}\|\mathbf{u}(t, \cdot)\|_{X_{\alpha}}+\sup _{(x, y) \in \mathbb{R}_{-}^{2}}[\mathbf{u}(\cdot, x, y)]_{C^{\alpha / 2}([a, b])}, \\
\|\mathbf{u}\| \mathcal{X}_{1+\alpha / 2,2+\alpha}(a, b)=\sum_{2 \alpha_{1}+\alpha_{2}+\alpha_{3} \leqslant 2}\left\|D_{t}^{\alpha_{1}} D_{x}^{\alpha_{2}} D_{y}^{\alpha_{3}} \mathbf{u}\right\| \mathcal{X}_{\alpha / 2, \alpha}(a, b) .
\end{gathered}
$$

Definition 3.2 For any $\alpha \in(0,1)$ and any $a, b \in \mathbb{R}_{+} \cup\{+\infty\}, a<b, C^{(j+\alpha) / 2, j+\alpha}([a, b] \times \mathbb{R})$ $(j=1,2)$ denotes the usual parabolic Hölder space

$$
\begin{aligned}
C^{(j+\alpha) / 2, j+\alpha}([a, b] \times \mathbb{R})=\left\{\psi: \psi(t, \cdot) \in C_{b}^{j+\alpha}(\mathbb{R}), \sup _{t \in[a, b]}\|\psi(t, \cdot)\|_{C_{b}^{j+\alpha}(\mathbb{R})}<+\infty,\right. \\
\left.\psi(\cdot, y) \in C^{(j+\alpha) / 2}([a, b]), \sup _{y \in \mathbb{R}}\|\psi(\cdot, y)\|_{C^{(j+\alpha) / 2}([a, b])}<+\infty\right\},
\end{aligned}
$$

endowed with the norm

$$
\|\psi\|_{C^{(j+\alpha) / 2, j+\alpha}([a, b] \times \mathbb{R})}=\sup _{t \in[a, b]}\|\psi(t, \cdot)\|_{C_{b}^{j+\alpha}(\mathbb{R})}+\sup _{y \in \mathbb{R}}[\psi(\cdot, y)]_{C^{(j+\alpha) / 2}([a, b])}, \quad j=1,2 .
$$

Definition 3.3 We denote by $\mathcal{X}_{q}$ the Banach space of all functions $q: \overline{\mathbb{R}}_{+} \times \mathbb{R} \rightarrow \mathbb{R}$ such that $t \mapsto q(t, \cdot)$ is continuous in $[0,+\infty)$ with values in $C_{b}^{2}(\mathbb{R})$, is continuously differentiable with values in $C_{b}(\mathbb{R})$, bounded with values in $C_{b}^{2+\alpha}(\mathbb{R})(\alpha \in(0,1)) ; t \mapsto q_{t}(t, \cdot)$ and $t \mapsto q_{y y}(t, \cdot)$ are Hölder continuous with exponent $\alpha / 2$ and with values in $C_{b}(\mathbb{R}) ; q_{t}$ is bounded with values in $C_{b}^{\alpha}(\mathbb{R})$ and

$$
\begin{aligned}
\|q\| \mathcal{X}_{q}= & \sup _{t \geqslant 0}\|q(t, \cdot)\|_{C_{b}(\mathbb{R})}+\sup _{t \geqslant 0}(1+t)\left(\left\|q_{t}(t, \cdot)\right\|_{C_{b}^{\alpha}(\mathbb{R})}+\left\|q_{y y}(t, \cdot)\right\|_{C_{b}^{\alpha}(\mathbb{R})}\right) \\
& +\sup _{0 \leqslant s<t}(1+s)\left(\frac{\left\|q_{t}(t, \cdot)-q_{t}(s, \cdot)\right\|_{C_{b}(\mathbb{R})}}{(t-s)^{\alpha / 2}}+\frac{\left\|q_{y y}(t, \cdot)-q_{y y}(s, \cdot)\right\|_{C_{b}(\mathbb{R})}}{(t-s)^{\alpha / 2}}\right)<+\infty .
\end{aligned}
$$

Moreover, we denote by $\mathcal{X}_{\mathbf{w}}$ the space of all functions $\mathbf{w}: \overline{\mathbb{R}}_{+} \times \overline{\mathbb{R}}_{-}^{2} \rightarrow \mathbb{R}^{3}$ such that $t \mapsto$ $\mathbf{w}(t, \cdot)$ is continuous in $[0,+\infty)$ with values in $X_{2}$, is continuously differentiable with values in $X_{0}$, bounded with values in $X_{2+\alpha} ; t \mapsto D_{t} \mathbf{w}$ and $t \mapsto \mathbf{w}(t, \cdot)$ are Hölder continuous with exponent $\alpha / 2$ and with values in $X_{0}$ and $X_{2}$, respectively, and

$$
\begin{aligned}
\|\mathbf{w}\|_{\mathcal{X}_{\mathbf{w}}}= & \sup _{t \geqslant 0}(1+t)\left(\|\mathbf{w}(t, \cdot)\|_{X_{2+\alpha}}+\left\|\mathbf{w}_{t}(t, \cdot)\right\|_{X_{\alpha}}\right) \\
& +\sup _{0 \leqslant s<t}(1+s)\left(\frac{\|\mathbf{w}(t, \cdot)-\mathbf{w}(s, \cdot)\|_{X_{2}}}{(t-s)^{\alpha / 2}}+\frac{\left\|D_{t} \mathbf{w}(t, \cdot)-D_{t} \mathbf{w}(s, \cdot)\right\|_{X_{0}}}{(t-s)^{\alpha / 2}}\right)<+\infty .
\end{aligned}
$$

We conclude this section with the following lemma.

LEMMA 3.4 Let $q: \overline{\mathbb{R}}_{+} \times \mathbb{R} \rightarrow \mathbb{R}$ be a continuous function such that

$$
[[q]]:=\sup _{t \geqslant 0}\|q(t, \cdot)\|_{C_{b}(\mathbb{R})}+\sup _{t \geqslant 0}(1+t)\left(\left\|q_{y y}(t, \cdot)\right\|_{C_{b}^{\alpha}(\mathbb{R})}+\left\|q_{t}(t, \cdot)\right\|_{C_{b}^{\alpha}(\mathbb{R})}\right)<+\infty .
$$


Then

$\begin{cases}\text { (i) } & \sup _{t \geqslant 0}(1+t)^{1 / 2}\left\|q_{y}(t, \cdot)\right\|_{C_{b}(\mathbb{R})} \leqslant C[[q]] ; \\ & \text { (ii) } \sup _{0 \leqslant s<t}(1+s)(t-s)^{-(2-j+\alpha) / 2}\left\|D_{y}^{j} q(t, \cdot)-D_{y}^{j} q(s, \cdot)\right\|_{C_{b}(\mathbb{R})} \leqslant C[[q]], \quad j=1,2 .\end{cases}$

If $\mathbf{w}: \overline{\mathbb{R}}_{+} \times \overline{\mathbb{R}}_{-} \times \mathbb{R} \rightarrow \mathbb{R}$ is such that

$$
[[\mathbf{w}]]:=\sup _{t \geqslant 0}(1+t)\left(\|\mathbf{w}(t, \cdot)\|_{X_{2+\alpha}}+\left\|D_{t} \mathbf{w}(t, \cdot)\right\|_{X_{\alpha}}\right)<+\infty,
$$

then

$$
\sup _{0 \leqslant s<t}(1+s)(t-s)^{-(2-j+\alpha) / 2}\|\mathbf{w}(t, \cdot)-\mathbf{w}(s, \cdot)\|_{X_{j}} \leqslant C[[\mathbf{w}]], \quad j=1,2 .
$$

Here $C$ denotes a positive constant independent of $q$ (resp. of $\mathbf{w})$.

Proof. The proof is based on interpolation inequalities. Estimates 3.1 ) and (3.1ii), for $j=1$, have been proved in [2, Lemma 2.5]. To prove (3.1 i) with $j=2$ and (3.2) it suffices to argue as in the proof of the quoted lemma, observing that, for any $\alpha \in(0,1)$, there exists a positive constant $C$ such that

$$
\left\|f^{\prime \prime}\right\|_{C_{b}(\mathbb{R})} \leqslant C[f]_{C_{b}^{\alpha}(\mathbb{R})}^{\alpha / 2}\left[f^{\prime \prime}\right]_{C_{b}^{\alpha}(\mathbb{R})}^{1-\alpha / 2}, \quad \forall f \in C_{b}^{2+\alpha}(\mathbb{R})
$$

and

$$
\|\mathbf{u}\|_{X_{j}} \leqslant C\|\mathbf{u}\|_{X_{\alpha}}^{(2-j+\alpha) / 2}\|\mathbf{u}\|_{X_{2+\alpha}}^{(j-\alpha) / 2}, \quad \forall \mathbf{u} \in X_{2+\alpha}, \quad j=1,2
$$

\section{The fully nonlinear problem}

This section, the main body of the paper, is devoted to proving that the null solution to problem 2.15 is stable with respect to $X_{2+\alpha}$-perturbations. As we are going to show, we are in a critical case of stability, since the spectrum of the realization $L$ of $\mathcal{L}$ in $X_{0}$ is contained in the left half-plane and contains 0 as an accumulation point of eigenvalues. Hence, we cannot apply the linearized stability principle to prove our stability results, since we cannot eliminate the eigenvalue 0 from the spectrum of $L$ by a spectral projection. Nevertheless we can define a suitable projection $P$ which is not a spectral projection, but enjoys most of the typical properties of a spectral projection. In particular (see Theorem 4.3 , the restriction of $e^{t L}$ to $(I-P)\left(X_{0}\right)$ gives rise to an (analytic) semigroup of negative type.

To get such a projection we observe that the operator $L$ can be split into the sum of two operators $L_{1}$ and $L_{2}$ commuting in the resolvent set. 0 is a simple eigenvalue of $L_{1}$. Hence, we can define a spectral projection associated with it: this will be our projection $P$.

\subsection{The realization of $\mathcal{L}$ in weighted Hölder spaces}

In this subsection we show that the realization $L$ of the differential operator $\mathcal{L}$ in $X_{0}$ generates an analytic semigroup, we characterize its domain and spectrum, and the interpolation spaces of order $\alpha / 2,1 / 2$ and $1+\alpha / 2(\alpha \in(0,1))$. Finally we provide a suitable projection $P$ satisfying (1.4).

To begin with, let us consider the following theorem which has been already proved, in a more general context, in [7]. 
THEOREM 4.1 The realization $L$ of the operator $\mathcal{L}$ in $X_{0}$ generates an analytic semigroup provided we set

$$
D(L)=\left\{\mathbf{u} \in X_{0} \cap \bigcap_{1<q<+\infty} W_{\mathrm{loc}}^{2, q}\left(\overline{\mathbb{R}}_{-}^{2}\right): \mathcal{L} \mathbf{u} \in X_{0}, \mathcal{B} \mathbf{u}(0, y)=\mathbf{0} \forall y \in \mathbb{R}\right\},
$$

where the boundary differential operator $\mathcal{B}$ is given by 2.9). Its spectrum is given by $\sigma(L)=$ $(-\infty, 0]$. Moreover, for every $\alpha \in(0,1)$ the set equalities

$$
\begin{aligned}
D_{L}(\alpha / 2, \infty) & =\left\{\mathbf{u} \in X_{\alpha}: B_{0} \mathbf{u}=0 \text { at } x=0\right\}, \\
D_{L}(1+\alpha / 2, \infty) & =\left\{\mathbf{u} \in X_{2+\alpha}: \mathcal{B} \mathbf{u}=\mathbf{0}, B_{0} L \mathbf{u}=0 \text { at } x=0\right\}
\end{aligned}
$$

hold, with equivalence of the respective norms. Finally,

$$
\left\{\mathbf{u} \in X_{1}: B_{0} \mathbf{u}=0 \text { at } x=0\right\} \subset D_{L}(1 / 2, \infty),
$$

with continuous embedding.

Proof. See [7, Theorem A.2 \& A.3].

As Theorem 4.1 shows we are in a critical case of stability, since the spectrum of $L$ is contained in the left half-plane and 0 is an accumulation point of eigenvalues. To construct a suitable projection satisfying (1.4) we begin by splitting operator $\mathcal{L}$ into the sum of the two operators $L_{1}: D\left(L_{1}\right) \subset$ $X_{0} \rightarrow X_{0}$ and $L_{2}: D\left(L_{2}\right) \subset X_{0} \rightarrow X_{0}$ formally defined by $L_{1} \mathbf{u}=\left(v_{x x}-v_{x}, w_{x x}-w_{x}, h_{x x}+h_{x}\right)$ and $L_{2} \mathbf{u}=\left(v_{y y}, w_{y y}, h_{y y}\right)$, where

$$
\begin{aligned}
& D\left(L_{1}\right)=\left\{\mathbf{u}: D_{x}^{j} \mathbf{u} \in X_{0} \text { for } j=0,1,2, \mathcal{B} \mathbf{u}=\mathbf{0}\right\} \\
& D\left(L_{2}\right)=\left\{\mathbf{u}: D_{y}^{j} \mathbf{u} \in X_{0} \text { for } j=0,1,2\right\} .
\end{aligned}
$$

Let us consider the following lemma.

LEMMA 4.2 Both the operators $L_{1}$ and $L_{2}$ are infinitesimal generators of analytic semigroups in $X_{0}$ provided their domains are chosen as in 4.2$) ; \sigma\left(L_{1}\right)=(-\infty,-1 / 4] \cup\{0\}$ and $\sigma\left(L_{2}\right)=$ $(-\infty, 0]$. Moreover, for any $\mathbf{f}=(f, g, k) \in X_{0}$,

$$
\begin{aligned}
{\left[R\left(\omega, L_{1}\right) \mathbf{f}\right]_{1} } & =\frac{1}{\sqrt{1+4 \omega}}\left(a(\mathbf{f}) e^{\mu_{2} x}-\int_{0}^{x} e^{\mu_{2} t} f(x-t, y) \mathrm{d} t+\int_{-\infty}^{0} e^{-\mu_{1} t} f(t+x, y) \mathrm{d} t\right), \\
{\left[R\left(\omega, L_{1}\right) \mathbf{f}\right]_{2} } & =\frac{1}{\sqrt{1+4 \omega}}\left(b(\mathbf{f}) e^{\mu_{2} x}-\int_{0}^{x} e^{\mu_{2} t} g(x-t, y) \mathrm{d} t+\int_{-\infty}^{0} e^{-\mu_{1} t} g(t+x, y) \mathrm{d} t\right), \\
{\left[R\left(\omega, L_{1}\right) \mathbf{f}\right]_{3} } & =\frac{1}{\sqrt{1+4 \omega}}\left(c(\mathbf{f}) e^{-\mu_{1} x}-\int_{0}^{x} e^{-\mu_{1} t} k(x-t, y) \mathrm{d} t+\int_{-\infty}^{0} e^{\mu_{2} t} k(t+x, y) \mathrm{d} t\right),
\end{aligned}
$$

where $\mu_{j}=\frac{1}{2}+(-1)^{j} \frac{1}{2} \sqrt{1+4 \omega}$ for $j=1,2$ and

$$
\begin{aligned}
& a(\mathbf{f})=\frac{1}{\mu_{2}-1}\left(\mu_{2} \int_{-\infty}^{0} e^{-\mu_{1} t} f(t, y) \mathrm{d} t+\int_{-\infty}^{0} e^{-\mu_{1} t} g(t, y) \mathrm{d} t+\int_{-\infty}^{0} e^{\mu_{2} t} k(t, y) \mathrm{d} t\right), \\
& b(\mathbf{f})=\int_{-\infty}^{0} e^{\mu_{2} t} k(t, y) \mathrm{d} t, \quad c(\mathbf{f})=\int_{-\infty}^{0} e^{-\mu_{1} t} g(t, y) \mathrm{d} t .
\end{aligned}
$$


Finally, $\omega=0$ is a simple eigenvalue of $L_{1}$; the corresponding eigenspace is spanned by the function $\mathbf{U}_{0}=\left(\Theta^{0}, 0,0\right)$ and the spectral projection associated with it is the operator $P: X_{0} \rightarrow X_{0}$ defined by $P \mathbf{u}=M(\mathbf{u}) \mathbf{U}_{0}$, where

$$
M(\mathbf{u})(y)=\int_{-\infty}^{0} v(x, y) \mathrm{d} x+\int_{-\infty}^{0} w(x, y) \mathrm{d} x+\int_{-\infty}^{0} e^{x} h(x, y) \mathrm{d} x, \quad \forall y \in \mathbb{R} .
$$

Proof. The proof, being straightforward, is left to the reader.

We are now going to prove that, even if $P$ is not a spectral projection associated with the operator $L$, it enjoys most of the typical properties of a spectral projection. In particular, $P$ commutes with $e^{t L}$, and $(I-P) e^{t L}$ decays with exponential rate as $t$ tends to $+\infty$. This property will play a crucial role in proving our stability results.

TheOREM 4.3 The operator $P$ defined in Lemma 4.2 commutes with the semigroup $e^{t L}$. Moreover, for any $\varepsilon>0$ there exists a positive constant $M:=M(\varepsilon)$ such that

$$
\left\|(I-P) e^{t L}\right\|_{L\left(X_{0}\right)} \leqslant M e^{(-1 / 4+\varepsilon) t} .
$$

In particular, 1.4 holds true for any space and time derivative of $e^{t L}$.

Proof. We begin the proof by observing that $e^{t L}=e^{t L_{1}} e^{t L_{2}}$ for any $t \geqslant 0$. To check this property we can limit ourselves to proving that all the assumptions of Da Prato-Grisvard's Theorem (see [4]) are satisfied. Hence, we need to check that $L_{1}$ and $L_{2}$ commute in the resolvent sense. Of course, thanks to Lemma A.1, we can limit ourselves to showing that $L_{2} R\left(\omega, L_{1}\right) \mathbf{f}=R\left(\omega, L_{1}\right) L_{2} \mathbf{f}$ for any $\omega \in \rho\left(L_{1}\right)$ and any $\mathbf{f} \in D\left(L_{2}\right)$, and this follows immediately if we take (4.3)- 4.5) into account.

Now, recalling that $P$ is the spectral projection associated with the simple eigenvalue $\omega=0$ of the operator $L_{1}$ we can write

$$
P \mathbf{u}=\frac{1}{2 \pi i} \int_{\gamma} R\left(\omega, L_{1}\right) \mathbf{u} \mathrm{d} \omega, \quad \forall \mathbf{u} \in X_{0},
$$

where $\gamma$ is a suitable closed and smooth curve around $\omega=0$ contained in $\rho\left(L_{1}\right)$, oriented counterclockwise.

Fix $\omega_{0} \in \rho\left(L_{2}\right)$. Applying $R\left(\omega_{0}, L_{2}\right)$ to both sides of 4.8$)$ and taking into account the fact that $L_{1}$ and $L_{2}$ commute in the resolvent sense, we can easily show that $P$ commutes with $R\left(\omega_{0}, L_{2}\right)$, and consequently it commutes with $e^{t L_{2}}$. This is enough for our aims. Indeed, $e^{t L}=e^{t L_{1}} e^{t L_{2}}$ for any $t>0$. Hence,

$$
P e^{t L}=P e^{t L_{1}} e^{t L_{2}}=e^{t L_{1}} P e^{t L_{2}}=e^{t L_{1}} e^{t L_{2}} P=e^{t L} P
$$

So that $P$ commutes with the semigroup $e^{t L}$. Moreover, since $\omega=0$ is an isolated simple eigenvalue of $L_{1}$ and $\sup \left\{\operatorname{Re} \omega: \omega \in \sigma\left(L_{1}\right), \omega \neq 0\right\}=-1 / 4$, it follows that for any $\varepsilon>0$ there exists a positive constant $M:=M(\varepsilon)$ such that

$$
\left\|(I-P) e^{t L_{1}}\right\|_{L\left(X_{0}\right)} \leqslant M e^{(\varepsilon-1 / 4) t}, \quad \forall t>0 .
$$

Since $e^{t L_{2}}$ is the heat semigroup, we have $\left\|e^{t L_{2}}\right\|_{L\left(X_{0}\right)} \leqslant 1$ for any $t \geqslant 0$. Hence,

$$
\left\|(I-P) e^{t L}\right\|_{L\left(X_{0}\right)}=\left\|(I-P) e^{t L_{1}} e^{t L_{2}}\right\|_{L\left(X_{0}\right)} \leqslant\left\|(I-P) e^{t L_{1}}\right\|_{L\left(X_{0}\right)}\left\|e^{t L_{2}}\right\|_{L\left(X_{0}\right)} \leqslant M e^{(\varepsilon-1 / 4) t}
$$


for any $t>0$, and 4.7 follows. Now observing that $L e^{t L}(I-P)=L e^{L} e^{(t-1) L}(I-P)$ for any $t>0$, we easily deduce that $L e^{t L}(I-P)$ decays at least as $e^{(\varepsilon-1 / 4) t}$ as $t$ tends to $+\infty$. The same holds for $L^{n} e^{t L}(I-P)$ for any $n \in \mathbb{N}$. If we take into account the characterization of the interpolation spaces $D_{L}(\alpha / 2, \infty)$ and $D_{L}(1+\alpha / 2, \infty)$ in Theorem 4.1. estimate 1.4 immediately follows.

\subsection{A suitable splitting of problem 2.15)}

In this subsection we deal with the initial value problem $\mathbf{u}(0, \cdot)=\mathbf{u}_{0}$ for the nonlinear problem 2.15). To begin with, we split $\mathbf{u}$ along $P\left(X_{0}\right)$ and $(I-P)\left(X_{0}\right)$ as $\mathbf{u}=P \mathbf{u}+\mathbf{w}:=r(t, y) \mathbf{U}_{0}+\mathbf{w}$. Then we determine the Cauchy problems satisfied by the pair $(r, \mathbf{w})$. For this purpose we begin by observing that $\mathcal{L} \mathbf{u}=\mathcal{L} P \mathbf{u}+\mathcal{L} \mathbf{w}$. Moreover, $\mathcal{L} P \mathbf{u}=r_{y y} \mathbf{U}_{0}$ and

$$
P \mathcal{L} \mathbf{v}=-\left(B_{2} \mathbf{v}\right) \mathbf{U}_{0}, \quad \forall \mathbf{v} \in(I-P)\left(X_{2}\right) \text { such that } B_{0} \mathbf{v}=B_{1} \mathbf{v}=0 .
$$

Hence, $r$ and $\mathbf{w}$ turn out to solve the following coupled Cauchy problems:

$$
\text { (i) }\left\{\begin{array} { l } 
{ r _ { t } = r _ { y y } - B _ { 2 } \mathbf { w } + M ( \mathcal { F } ( \mathbf { u } ) ) , } \\
{ r ( 0 , \cdot ) = M ( \mathbf { u } _ { 0 } ) , }
\end{array} \quad \text { (ii) } \quad \left\{\begin{array}{l}
D_{t} \mathbf{w}=\mathcal{L} \mathbf{w}+\left(B_{2} \mathbf{w}\right) \mathbf{U}_{0}+(I-P) \mathcal{F}(\mathbf{u}) \\
\mathcal{B} \mathbf{w}=\mathcal{G}(\mathbf{u}) \\
\mathbf{w}(0, \cdot)=(I-P) \mathbf{u}_{0}
\end{array}\right.\right.
$$

where the linear operator $M$ is defined in 4.6. Straightforward computations and the fact that $\mathcal{B} \mathbf{u}=\mathcal{B} \mathbf{w}=(0,0, g(\mathbf{u}))$ show that

$$
\begin{aligned}
M(\mathcal{F}(\mathbf{u}))= & \left(r_{y}+v_{y}(0)\right)^{2}\left(\frac{e^{h(0)}}{\sqrt{1+\left(r_{y}+v_{y}(0)\right)^{2}}}-h(0)+\frac{v_{x}(0)-v_{x x}(0)}{1-v(0)+v_{x}(0)} \int_{-\infty}^{0} e^{x} h(x) \mathrm{d} x\right) \\
& +2\left(r_{y}+v_{y}(0)\right)\left(\int_{-\infty}^{0} e^{x} h_{y}(x) \mathrm{d} x+\frac{v_{y}(0)-v_{x y}(0)}{1-v(0)+v_{x}(0)} \int_{-\infty}^{0} e^{x} h(x) \mathrm{d} x\right) \\
& -\frac{v_{x x}(0)-v_{x}(0)}{1-v(0)+v_{x}(0)} \int_{-\infty}^{0} e^{x} h(x) \mathrm{d} x,
\end{aligned}
$$

where, to shorten the notation, we simply wrote $D^{\alpha} v(x), D^{\alpha} w(x)$ and $D^{\alpha} h(x)(|\alpha| \leqslant 2, x \leqslant 0)$ instead of $D^{\alpha} v(\cdot, x, \cdot), D^{\alpha} w(\cdot, x, \cdot)$ and $D^{\alpha} h(\cdot, x, \cdot)$.

Let us now consider the differential equation in 4.10 ). Since, as already pointed out, $B_{2} \mathbf{w}=$ $g(\mathbf{u})$, we can write

$$
r_{t}(t, y)=r_{y y}(t, y)+\frac{1}{2} r_{y}^{2}+\mathcal{H}(r(t, \cdot), \mathbf{w}(t, \cdot))(y), \quad \forall y \in \mathbb{R}
$$

where $\mathcal{H}(r, \mathbf{w})$ is a nonlinear operator which is quadratic near $(0, \mathbf{0})$. The second order terms in the expression of $\mathcal{H}$ are both quadratic in the derivatives of $\mathbf{w}$, and are given by the product of $\left(\partial^{j} / \partial y^{j}\right) r$ $(j=0,1,2)$ multiplied either by $y$-derivatives of $v$ and $h$ or by $\int_{-\infty}^{0} e^{x} h(x) \mathrm{d} x$. Equation 4.11) exhibits a critical growth at 0 due to the presence of the term $\frac{1}{2} r_{y}^{2}$. To skip the problems given by the nonlinearity, we perform a Cole-Hopf transformation, namely we set $q:=e^{r / 2}-1$. Straightforward 
computations show that the differential equation 4.11) transforms into the differential equation $q_{t}(t, y)=q_{y y}(t, y)+\widetilde{\mathcal{H}}(q(t, \cdot), \mathbf{w}(t, \cdot))(y)$ for the unknown $(q, \mathbf{w})$, where

$$
\begin{aligned}
& \tilde{\mathcal{H}}(q, \mathbf{w})=\frac{q+1}{2}\left(\frac{2 q_{y}}{q+1}+v_{y}(0)\right)^{2} \\
& \times\left(\frac{e^{h(0)}}{\sqrt{1+\left(\frac{2 q_{y}}{q+1}+v_{y}(0)\right)^{2}}}-h(0)-1+\frac{v_{x}(0)-v_{x x}(0)}{1-v(0)+v_{x}(0)} \int_{-\infty}^{0} e^{x} h(x) \mathrm{d} x\right) \\
& +2\left(q_{y}+\frac{q+1}{2} v_{y}(0)\right)\left(\int_{-\infty}^{0} e^{x} h_{y}(x) \mathrm{d} x+\frac{v_{y}(0)-v_{x y}(0)}{1-v(0)+v_{x}(0)} \int_{-\infty}^{0} e^{x} h(x) \mathrm{d} x\right) \\
& -\frac{(q+1)\left(v_{x x}(0)-v_{x}(0)\right)}{2\left(1-v(0)+v_{x}(0)\right)} \int_{-\infty}^{0} e^{x} h(x) \mathrm{d} x+\frac{q+1}{4}\left(v_{y}(0)\right)^{2}+q_{y} v_{y}(0) \\
& +\frac{q+1}{2}\left(e^{h(0)}-1\right)\left(\frac{1}{\sqrt{1+\left(\frac{2 q_{y}}{q+1}+v_{y}(0)\right)^{2}}}-1\right)+\frac{q+1}{2}\left(e^{h(0)}-1-h(0)\right) \\
& +\frac{q+1}{2}\left(\frac{1}{\sqrt{1+\left(\frac{2 q_{y}}{q+1}+v_{y}(0)\right)^{2}}}-1+\frac{1}{2}\left(\frac{2 q_{y}}{q+1}+v_{y}(0)\right)^{2}\right) \text {. }
\end{aligned}
$$

Let us now observe that

$$
\mathcal{K}(q, \mathbf{w}):=B_{2} \mathbf{w}+(I-P) \mathcal{F}(\mathbf{u}):=\tilde{g}(q, \mathbf{w}) \mathbf{U}_{0}+(I-P)\left(\mathcal{F}\left(2 \log (q+1) \mathbf{U}_{0}+\mathbf{w}\right)\right),
$$

where

$$
\begin{gathered}
\tilde{g}(q, \mathbf{w})=1+h(0)-\frac{e^{h(0)}}{\sqrt{1+\left(\frac{2 q_{y}}{q+1}+v_{y}(0)\right)^{2}}} ; \\
(I-P) \mathcal{F}\left(2 \log (q+1) \mathbf{U}_{0}+\mathbf{w}\right) \\
=\left(\frac{2 q_{y}}{q+1}+v_{y}(0)\right)^{2}\left[\mathbf{w}_{x x}-\frac{v_{x x}(0)-v(0)+1}{1-v(0)+v_{x}(0)} \widetilde{\mathbf{w}}_{x}+\left(1+\frac{v_{x x}(0)-v_{x}(0)}{1-v(0)+v_{x}(0)} v(0)\right) \mathbf{U}_{0}\right. \\
-\left(\frac{e^{h(0)}}{\left.\left.\sqrt{1+\left(\frac{2 q_{y}}{q+1}+v_{y}(0)\right)^{2}}-h(0)-\frac{v_{x x}(0)-v_{x}(0)}{1-v(0)+v_{x}(0)} \int_{-\infty}^{0} e^{x} h(x) \mathrm{d} x\right) \mathbf{U}_{0}\right]}\right] \\
+2\left(\frac{2 q_{y}}{q+1}+v_{y}(0)\right)\left[\widetilde{\mathbf{w}}_{x y}-\frac{v_{x y}(0)-v_{y}(0)}{1-v(0)+v_{x}(0)} \widetilde{\mathbf{w}}_{x}-\frac{v_{y}(0)-v_{x y}(0)}{1-v(0)+v_{x}(0)}\left(\int_{-\infty}^{0} e^{x} h(x) \mathrm{d} x\right) \mathbf{U}_{0}\right. \\
-\frac{v_{x x}(0)-v_{x}(0)}{1-v(0)+v_{x}(0)}\left[\widetilde{\mathbf{w}}_{x}-\left(\int_{-\infty}^{0} e^{x} h_{y}(x) \mathrm{d} x\right) \mathbf{U}_{0}+\frac{v(0) v_{x y}(0)-v_{y}(0)\left(1+v_{x}(0)\right)}{1-v(0)+v_{x}(0)} \mathbf{U}_{0}\right]
\end{gathered}
$$


and $\widetilde{\mathbf{w}}=(v, w,-h)$. Hence, the pair $(q, \mathbf{w})$ turns out to solve the Cauchy problems

$$
\text { (i) }\left\{\begin{array} { l } 
{ q _ { t } = q _ { y y } + \tilde { \mathcal { H } } ( q , \mathbf { w } ) , } \\
{ q ( 0 , \cdot ) = \operatorname { e x p } ( M ( \mathbf { u } _ { 0 } ) / 2 ) - 1 , }
\end{array} \quad \text { (ii) } \left\{\begin{array}{l}
D_{t} \mathbf{w}=\mathcal{L} \mathbf{w}+\mathcal{K}(q, \mathbf{w}) \\
\mathcal{B} \mathbf{w}=\mathcal{G}(\mathbf{u}) \\
\mathbf{w}(0, \cdot)=(I-P) \mathbf{u}_{0}
\end{array}\right.\right.
$$

where the nonlinear operators $\widetilde{\mathcal{H}}$ and $\mathcal{K}$ are given, respectively, by 4.12 and $4.13-4.15$.

\subsection{Optimal regularity for the linear problem}

We devote this section to proving optimal Schauder estimates for the solutions to the linearized problems associated with systems 4.16, namely the problems

$$
\text { (i) }\left\{\begin{array} { l } 
{ q _ { t } = q _ { y y } + \varphi , } \\
{ q ( 0 , \cdot ) = q _ { 0 } , }
\end{array} \quad \text { (ii) } \left\{\begin{array}{l}
D_{t} \mathbf{w}=\mathcal{L} \mathbf{w}+\mathbf{f}, \\
\mathcal{B} \mathbf{w}=(0,0, \psi), \\
\mathbf{w}(0, \cdot)=\mathbf{w}_{0}
\end{array}\right.\right.
$$

Problem (4.17) has been partly already considered in [2, Theorem 2.6]. Note that the asymptotic estimates of the solution to problem 4.16 are crucial to proving our stability results. However, since not all the estimates we need are contained in the quoted theorem, we give the details.

THEOREM 4.4 Let $\varphi: \overline{\mathbb{R}}_{+} \times \mathbb{R} \rightarrow \mathbb{R}$ satisfy

$$
[[\varphi]]_{0}=\sup _{t \geqslant 0}\left(1+t^{1+\alpha / 2}\right)\|\varphi(t, \cdot)\|_{C_{b}^{\alpha}(\mathbb{R})}+\sup _{0 \leqslant s<t}(1+s) \frac{\|\varphi(t, \cdot)-\varphi(s, \cdot)\|_{C_{b}(\mathbb{R})}}{(t-s)^{\alpha / 2}}<+\infty
$$

for some $\alpha \in(0,1)$. Further, assume that $q_{0} \in C_{b}^{2+\alpha}(\mathbb{R})$. Then problem 4.17 ) admits a unique bounded strict solution $q$, which belongs to $\mathcal{X}_{q}$ (see Definition 3.3) and satisfies the following estimate:

$$
\|q\| \mathcal{X}_{q}+\sup _{s \geqslant 0}(1+s) \frac{\left\|q_{y}(t, \cdot)-q_{y}(s, \cdot)\right\|_{C_{b}(\mathbb{R})}}{(t-s)^{(1+\alpha) / 2}} \leqslant C\left(\left\|q_{0}\right\|_{C_{b}^{2+\alpha}(\mathbb{R})}+[[\varphi]]_{0}\right),
$$

for some positive constant $C$, independent of $\left(q_{0}, \varphi\right)$.

Proof. Throughout the proof, we denote by $C_{j}(j \in \mathbb{N})$ positive constants independent of the data and $t$.

As is well known (see e.g. [10, Theorems 4.3.1 \& 4.3.8]), our assumptions guarantee that problem 4.17) admits a unique strict solution $q \in C^{1+\alpha / 2}\left([0, T] ; C_{b}(\mathbb{R})\right) \cap B\left([0, T] ; C_{b}^{2+\alpha}(\mathbb{R})\right) \cap$ $C^{\alpha / 2}\left([0, T] ; C_{b}^{2}(\mathbb{R})\right)$ such that $q_{t} \in B\left([0, T] ; C_{b}^{\alpha}(\mathbb{R})\right)$ for any $T>0$. The solution $q$ is given by the variation-of-constants formula

$$
q(t, \cdot)=T(t) q_{0}+\int_{0}^{t} T(t-s) \varphi(s, \cdot) \mathrm{d} s, \quad \forall t \in[0, T],
$$

where $T(t)$ is the Gauss-Weierstrass semigroup, i.e.

$$
(T(t) g)(y)=\frac{1}{\sqrt{4 \pi t}} \int_{\mathbb{R}} e^{-|y-z|^{2} / 4 t} g(z) \mathrm{d} z, \quad \forall t>0, y \in \mathbb{R},
$$


for any $g \in C_{b}(\mathbb{R})$. Moreover, there exists a positive constant $C_{1}$ such that

$$
\begin{aligned}
\|q\|_{B\left([0,1] ; C_{b}^{2+\alpha}(\mathbb{R})\right)}+\left\|q_{t}\right\|_{B\left([0,1] ; C_{b}^{\alpha}(\mathbb{R})\right)}+\left\|q_{t}\right\|_{C^{\alpha / 2}\left([0,1] ; C_{b}(\mathbb{R})\right)} & +\left\|q_{y y}\right\|_{C^{\alpha / 2}\left([0,1] ; C_{b}(\mathbb{R})\right)} \\
& \leqslant C_{1}\|\varphi\|_{B\left([0,1] ; C_{b}^{\alpha}(\mathbb{R})\right) .}
\end{aligned}
$$

It is also well known that for any $\beta \in[0,1)$ and any $k \in \mathbb{N}$ there exist positive constants $C_{\beta, k}$ such that

$$
\left\|D_{y}^{k} T(t) g\right\|_{C_{b}(\mathbb{R})} \leqslant C_{\beta, k} t^{-(k-\beta) / 2}\|g\|_{C_{b}^{\beta}(\mathbb{R})}, \quad \forall t>0, \quad \forall g \in C_{b}^{\beta}(\mathbb{R}) .
$$

It immediately follows that $T(\cdot) q_{0}$ belongs to $B\left([0,+\infty) ; C_{b}^{2+\alpha}(\mathbb{R})\right) \cap C_{b}^{1}\left([0,+\infty) ; C_{b}(\mathbb{R})\right)$ and there exists a positive constant $C_{2}$ such that

$$
\sup _{t \geqslant 0}\left\|T(t) q_{0}\right\|_{C_{b}(\mathbb{R})}+\sup _{t \geqslant 0}(1+t)\left\|D_{y y} T(t) q_{0}\right\|_{C_{b}^{\alpha}(\mathbb{R})} \leqslant C_{2}\left\|q_{0}\right\|_{C_{b}^{2+\alpha}(\mathbb{R})} .
$$

Let us now consider the convolution term $q_{1}(t, \cdot)=\int_{0}^{t} T(t-s) \varphi(s, \cdot) \mathrm{d} s$ and let us estimate $q_{1}$ for $t>1$. From (4.21) we immediately deduce that

$$
\left\|q_{1}(t, \cdot)\right\|_{C_{b}(\mathbb{R})} \leqslant \sup _{r \geqslant 0}\left(1+r^{1+\alpha / 2}\right)\|\varphi(r, \cdot)\|_{C_{b}(\mathbb{R})} \int_{0}^{+\infty}\left(1+s^{1+\alpha / 2}\right)^{-1} \mathrm{~d} s, \quad \forall t>1,
$$

since $C_{0,0}=1$. As far as the second order space derivative of $q_{1}$ is concerned, we observe that

$$
\begin{aligned}
\left\|D_{y}^{2} q_{1}(t, \cdot)\right\|_{C_{b}(\mathbb{R}) \leqslant} \leqslant & \left\|\int_{0}^{t / 2} D_{y}^{2} T(t-s) \varphi(s, \cdot) \mathrm{d} s\right\|_{C_{b}(\mathbb{R})}+\left\|\int_{t / 2}^{t} D_{y}^{2} T(t-s) \varphi(s, \cdot) \mathrm{d} s\right\|_{C_{b}(\mathbb{R})} \\
\leqslant & C_{0,2} \sup _{r \geqslant 0}\left(1+r^{1+\alpha / 2}\right)\|\varphi(r, \cdot)\|_{C_{b}(\mathbb{R})} \int_{0}^{t / 2} \frac{1}{1+s^{1+\alpha / 2}} \frac{1}{t-s} \mathrm{~d} s \\
& +C_{\alpha, 2} \sup _{r \geqslant 0}\left(1+r^{1+\alpha / 2}\right)\|\varphi(r, \cdot)\|_{C_{b}^{\alpha}(\mathbb{R})} \int_{t / 2}^{t} \frac{1}{1+s^{1+\alpha / 2}} \frac{1}{(t-s)^{1-\alpha / 2}} \mathrm{~d} s \\
\leqslant & C_{3} \sup _{r \geqslant 0}\left(1+r^{1+\alpha / 2}\right)\|\varphi(r, \cdot)\|_{C_{b}(\mathbb{R})} t^{-1}, \quad \forall t>1 .
\end{aligned}
$$

Moreover,

$$
\begin{aligned}
&\left\|\xi^{1-\alpha / 2} D_{y}^{2} T(\xi) D_{y}^{2} q_{1}(t, \cdot)\right\|_{C_{b}(\mathbb{R})}=\xi^{1-\alpha / 2}\left\|\int_{0}^{t} D_{y}^{4} T(t+\xi-s) \varphi(s, \cdot) \mathrm{d} s\right\|_{C_{b}(\mathbb{R})} \\
& \leqslant C_{\alpha, 4} \sup _{r \geqslant 0}\left(1+r^{1+\alpha / 2}\right)\|\varphi(r, \cdot)\|_{C_{b}^{\alpha}(\mathbb{R})} \int_{0}^{t / 2} \frac{\xi^{1-\alpha / 2}}{(t+\xi-s)^{2-\alpha / 2}} \frac{\mathrm{d} s}{1+s^{1+\alpha / 2}} \\
&+C_{\alpha, 4} \sup _{r \geqslant 0}\left(1+r^{1+\alpha / 2}\right)\|\varphi(r, \cdot)\|_{C_{b}^{\alpha}(\mathbb{R})} \int_{t / 2}^{t} \frac{\xi^{1-\alpha / 2}}{(t+\xi-s)^{2-\alpha / 2}} \frac{\mathrm{d} s}{1+s^{1+\alpha / 2}} \\
& \leqslant C_{\alpha, 4} \sup _{r \geqslant 0}\left(1+r^{1+\alpha / 2}\right)\|\varphi(r, \cdot)\|_{C_{b}^{\alpha}(\mathbb{R})}(t / 2)^{-(2-\alpha / 2)} \int_{0}^{+\infty} \frac{\mathrm{d} s}{1+s^{1+\alpha / 2}} \\
&+C_{\alpha, 4} \sup _{r \geqslant 0}\left(1+r^{1+\alpha / 2}\right)\|\varphi(r, \cdot)\|_{C_{b}^{\alpha}(\mathbb{R})}(t / 2)^{-(1+\alpha / 2)} \int_{0}^{+\infty} \frac{\mathrm{d} s}{(1+s)^{2-\alpha / 2}} \\
& \leqslant C_{4} t^{-(1+\alpha / 2)} \sup _{r \geqslant 0}\left(1+r^{1+\alpha / 2}\right)\|\varphi(r, \cdot)\|_{C_{b}^{\alpha}(\mathbb{R}), \quad \forall \xi \in(0,1) .}
\end{aligned}
$$


Hence,

$$
\begin{aligned}
& \sup _{\xi \in(0,1)}\left\|\xi^{1-\alpha / 2} D_{y}^{2} T(\xi) D_{y}^{2} q_{1}(t, \cdot)\right\|_{C_{b}(\mathbb{R})} \\
& \leqslant C_{4} t^{-(1+\alpha / 2)} \sup _{r \geqslant 0}\left(1+r^{1+\alpha / 2}\right)\|\varphi(r, \cdot)\|_{C_{b}^{\alpha}(\mathbb{R})}, \quad \forall t>1 .
\end{aligned}
$$

Since $f \in C_{b}^{\alpha}(\mathbb{R})$ if and only if $f \in C_{b}(\mathbb{R})$ and $[[f]]_{C_{b}^{\alpha}(\mathbb{R})}:=\sup _{\xi \in(0,1)}\left\|\xi^{1-\alpha / 2} D_{y}^{2} T(\xi) f\right\|_{C_{b}(\mathbb{R})}<$ $+\infty$, and the usual norm of $C_{b}^{\alpha}(\mathbb{R})$ is equivalent to the norm $\|\cdot\|_{C_{b}(\mathbb{R})}+[[\cdot]]_{C_{b}^{\alpha}(\mathbb{R})}$, from $(4.24)$ and 4.25 we immediately deduce that

$$
\sup _{t \geqslant 1}(1+t)\left\|D_{y}^{2} q_{1}(t, \cdot)\right\|_{C_{b}^{\alpha}(\mathbb{R})} \leqslant C_{5}\left(\left\|q_{0}\right\|_{C_{b}^{2+\alpha}(\mathbb{R})}+[[\varphi]]_{0}\right) .
$$

Taking 4.20, 4.22, 4.23, 4.26 and Lemma A.3 into account, we can now easily show that

$$
\sup _{t \geqslant 0}\|q(t, \cdot)\|_{C_{b}(\mathbb{R})}+\sup _{t \geqslant 0}(1+t)\left\|q_{y y}(t, \cdot)\right\|_{C_{b}^{\alpha}(\mathbb{R})} \leqslant C_{6}\left(\left\|q_{0}\right\|_{C_{b}^{2+\alpha}(\mathbb{R})}+[[\varphi]]_{0}\right) .
$$

Moreover, since $q_{t}(t, \cdot)=q_{y y}(t, \cdot)+\varphi(t, \cdot)$, from 4.27) and our assumptions on $\varphi$, we easily deduce that

$$
\sup _{t \geqslant 0}(1+t)\left\|D_{t} q(t, \cdot)\right\|_{C_{b}^{\alpha}(\mathbb{R})} \leqslant C_{7}\left(\left\|q_{0}\right\|_{C_{b}^{2+\alpha}(\mathbb{R})}+[[\varphi]]_{0}\right) .
$$

From 4.27, 4.28 and Lemma 3.4 we immediately deduce that $D_{y}^{j} q \in C_{b}^{(2-j+\alpha) / 2}([0,+\infty)$; $\left.C_{b}(\mathbb{R})\right)$ for $j=1,2$ and there exists a positive constant $C_{8}$ such that

$$
\begin{array}{r}
\sup _{0 \leqslant s<t}(1+s)\left(\frac{\left\|D_{y}^{j} q(t, \cdot)-D_{y}^{j} q(s, \cdot)\right\|_{C_{b}(\mathbb{R})}}{(t-s)^{(2-j+\alpha) / 2}}+\frac{\left\|D_{t} q(t, \cdot)-D_{t} q(s, \cdot)\right\|_{C_{b}(\mathbb{R})}}{(t-s)^{\alpha / 2}}\right) \\
\leqslant C_{8}\left(\left\|u_{0}\right\|_{C_{b}^{2+\alpha}(\mathbb{R})}+[[\varphi]]_{0}\right),
\end{array}
$$

for $j=1$, 2. Now, 4.19 follows from 4.27-4.29.

We now turn to problem 4.17ii). For this purpose we prove the following lemma which provides a suitable lifting operator mapping $C_{b}^{k}(\mathbb{R})$ into $X_{k+1}$ for any $k \geqslant 0$.

LEMmA 4.5 There exists a lifting operator $\mathcal{N} \in L\left(C_{b}^{k}(\mathbb{R}), X_{k+1}\right)$ for any $k \geqslant 0$ such that

(i) $\mathcal{B N} \psi=(0,0, \psi)$ for any $\psi \in C_{b}(\mathbb{R})$;

(ii) $P \mathcal{N} \psi=\mathbf{0}$ for any $\psi \in C_{b}(\mathbb{R})$;

(iii) $P \mathcal{L N} \psi=-\psi \mathbf{U}_{0}$ for any $\psi \in C_{b}^{1}(\mathbb{R})$.

Proof. Let $N$ be the linear operator defined by

$$
N \psi=x \eta(x) \int_{\mathbb{R}} \varphi(\xi) \psi(y+\xi x) \mathrm{d} \xi, \quad \forall \psi \in C_{b}(\mathbb{R}),
$$

where $\eta$ is any smooth function satisfying $\eta(x)=1$ for any $x \in[-1,0]$ and $\eta(x)=0$ for any $x \leqslant$ -2 , while $\varphi$ is any smooth even function compactly supported in $(-1,1)$ such that $0 \leqslant \varphi(x) \leqslant 1$ for any $x \in \mathbb{R}$ and with $\|\varphi\|_{L^{1}(\mathbb{R})}=1$. As is immediately seen, $N \in L\left(C_{b}^{k}(\mathbb{R}), C_{b}^{k+1, \sharp}\left(\overline{\mathbb{R}}_{-}^{2}\right)\right)$ for 
any $k \geqslant 0$, where $C_{b}^{k+1, \sharp}\left(\overline{\mathbb{R}}_{-}^{2}\right)$ denotes the set of all continuous functions $f: \overline{\mathbb{R}}_{-}^{2} \rightarrow \mathbb{R}$ such that $(x, y) \mapsto e^{-x / 2} f(x, y)$ is bounded. Moreover, $(N \psi)(0, \cdot)=0$ and $\left(D_{x} N \psi\right)(0, \cdot)=\psi$.

Define $\mathcal{N}_{0}$ by setting

$$
\mathcal{N} \psi=(I-P)(-N \psi, 0,0), \quad \forall \psi \in C_{b}(\mathbb{R}) .
$$

It is immediate to check that $\mathcal{N} \in L\left(C_{b}^{k}(\mathbb{R}), X_{k+1}\right)$ for any $k \geqslant 0$. Moreover, by construction, $\mathcal{N}$ has both properties (i) and (ii). Property (iii) easily follows from (i) and (4.9).

The solution to problem (4.17ii), under suitable assumptions on $\mathbf{w}_{0}, \mathbf{f}$ and $\psi$, is given by the following formula, a variant of the Balakrishnan formula:

$$
\begin{aligned}
\mathbf{w}(t, \cdot)= & e^{t L} \mathbf{w}_{0}+\int_{0}^{t} e^{(t-s) L}(\mathbf{f}(s, \cdot)+\mathcal{L N} \psi(s, \cdot)) \mathrm{d} s \\
& -L \int_{0}^{t} e^{(t-s) L} \mathcal{N} \psi(s, \cdot) \mathrm{d} s, \quad \forall t>0 .
\end{aligned}
$$

To be more precise, the following theorem holds.

TheOREM 4.6 Let $\mathbf{w}_{0} \in(I-P)\left(X_{2+\alpha}\right), \psi \in C^{(1+\alpha) / 2,1+\alpha}([0, T] \times \mathbb{R})$ and $\mathbf{f} \in \mathcal{X}_{\alpha / 2, \alpha}(0, T)$ $(T>0)$, with $\mathbf{f}(t, \cdot) \in(I-P)\left(X_{0}\right)$ for any $t \in[0, T]$, satisfy the compatibility conditions

$$
\mathcal{B} \mathbf{w}_{0}(0, \cdot)=(0,0, \psi(0, \cdot)), \quad B_{0}\left(\mathcal{L} \mathbf{w}_{0}(0, \cdot)+\mathbf{f}(0, \cdot)\right)=0 .
$$

Then problem 4.17 ii) admits a unique solution $\mathbf{w} \in \mathcal{X}_{1+\alpha / 2,2+\alpha}(0, T)$ given by 4.30 , where $\mathcal{N}$ is the lifting operator in Lemma 4.5 Moreover, $\mathbf{w}(t, \cdot) \in(I-P)\left(X_{0}\right)$ for any $t \in[0, T]$ and there exists a positive constant $C(T)>0$, independent of the data, such that

$$
\|\mathbf{w}\|_{\mathcal{X}_{1+\alpha / 2,2+\alpha}(0, T)} \leqslant C(T)\left(\left\|\mathbf{w}_{0}\right\|_{X_{2+\alpha}}+\|\mathbf{f}\|_{\mathcal{X}_{\alpha / 2, \alpha}(0, T)}+\|\psi\|_{C^{(1+\alpha) / 2,1+\alpha}([0, T] \times \mathbb{R})}\right) .
$$

Proof. The proof can be easily obtained by adapting the techniques of [7, Theorem 4.1].

Due to the particular nonlinearity $\widetilde{\mathcal{H}}$ we are considering, we can assume that $\mathbf{f}$ is split as $\mathbf{f}=\mathbf{g}+\psi \mathbf{U}_{0}$ for suitable functions $\mathbf{g}$ and $\psi$.

The following theorem deals with the asymptotic behaviour of the function $\mathbf{w}$ in 4.30 .

THEOREM 4.7 Suppose that $\mathbf{g} \in \mathcal{X}_{\alpha / 2, \alpha}(0, \infty)(\alpha \in(0,1))$ is such that $\mathbf{g}(t, \cdot) \in(I-P)\left(X_{\alpha}\right)$ for any $t \geqslant 0$ and

$$
[[\mathbf{g}]]_{1}:=\sup _{t \geqslant 0}(1+t)\|\mathbf{g}(t, \cdot)\|_{X_{\alpha}}+\sup _{0 \leqslant s<t}(1+s) \frac{\|\mathbf{g}(t, \cdot)-\mathbf{g}(s, \cdot)\|_{X_{0}}}{(t-s)^{\alpha / 2}}<+\infty .
$$

Further, assume that $\mathbf{w}_{0} \in(I-P)\left(X_{2+\alpha}\right)$ and $\psi \in C^{(1+\alpha) / 2,1+\alpha}([0,+\infty) \times \mathbb{R})$ is such that

$$
\begin{aligned}
{[[\psi]]_{2}=} & \sup _{t \geqslant 0}(1+t)\|\psi(t, \cdot)\|_{C_{b}^{1+\alpha}(\mathbb{R})} \\
& +\sup _{0 \leqslant s<t}(1+s)\left(\frac{\|\psi(t, \cdot)-\psi(s, \cdot)\|_{C_{b}(\mathbb{R})}}{(t-s)^{(1+\alpha) / 2}}+\frac{\left\|\psi_{y}(t, \cdot)-\psi_{y}(s, \cdot)\right\|_{C_{b}(\mathbb{R})}}{(t-s)^{\alpha / 2}}\right)<+\infty
\end{aligned}
$$


and

$$
\mathcal{B} \mathbf{w}_{0}=(0,0, \psi(0, \cdot)), \quad B_{0}\left(\mathcal{L} \mathbf{w}_{0}+\mathbf{g}(0, \cdot)\right)=(-\psi, 0,0) .
$$

Then problem (4.17 i) with $\mathbf{f}=\mathbf{g}+\psi \mathbf{U}_{0}$ admits a unique solution $\mathbf{w} \in \mathcal{X}_{\mathbf{w}}$ given by 4.30]. Moreover, $\mathbf{w}(t, \cdot) \in(I-P)\left(X_{0}\right)$ for any $t \geqslant 0$ and

$$
\|\mathbf{w}\|_{\mathcal{X}_{\mathbf{w}}}+\sup _{0 \leqslant s<t}(1+s) \frac{\|\mathbf{w}(t, \cdot)-\mathbf{w}(s, \cdot)\|_{X_{1}}}{(t-s)^{(1+\alpha) / 2}} \leqslant C\left(\left\|\mathbf{w}_{0}\right\|_{X_{2+\alpha}}+[[\mathbf{g}]]_{1}+[[\psi]]_{2}\right) .
$$

Before proving the theorem let us consider the following two lemmas.

Lemma 4.8 Let $Z$ be a Banach space, let $A: D(A) \subset Z \rightarrow Z$ be the generator of an analytic semigroup of negative type in $Z$, and let $u_{0} \in Z, f:[0,+\infty) \rightarrow Z$ be an $\alpha / 2$-Hölder continuous function such that

$$
[[f]]_{3}:=\sup _{t \geqslant 0}(1+t)\|f(t)\|_{Z}+\sup _{0 \leqslant s<t}(1+s) \frac{\|f(t, \cdot)-f(s, \cdot)\|_{Z}}{(t-s)^{\alpha / 2}}<+\infty,
$$

with $0<\alpha<1$. Then the function

$$
u(t)=e^{t A} u_{0}+\int_{0}^{t} e^{(t-s) A} f(s) \mathrm{d} s
$$

is the unique classical solution of $u^{\prime}(t)=A u(t)+f(t)$ such that $u(0)=u_{0} ; u^{\prime}(t, \cdot)$ has values in $D_{A}(\alpha / 2, \infty)$ for any $t>0$ and

$$
\begin{gathered}
\sup _{t \geqslant 1}(1+t)\left(\|u(t)\|_{D(A)}+\left\|u^{\prime}(t)\right\|_{D_{A}(\alpha / 2, \infty)}\right)+\sup _{1 \leqslant s<t}(1+s) \frac{\left\|u^{\prime}(t)-u^{\prime}(s)\right\|_{Z}}{(t-s)^{\alpha / 2}} \\
\leqslant C\left(\left\|u_{0}\right\|_{Z}+[[f]]_{3}\right) .
\end{gathered}
$$

Finally, if $u_{0} \in D(A)$ and $A u_{0}+f(0) \in D_{A}(\alpha / 2, \infty)$, then $u \in C^{1+\alpha / 2}([0,1] ; Z) \cap$ $C^{\alpha / 2}([0,1] ; D(A))$ and $u^{\prime} \in B\left([0,1] ; D_{A}(\alpha / 2, \infty)\right)$. Moreover, there exists a positive constant $C$, independent of the data, such that

$$
\begin{aligned}
\|u\|_{C^{1+\alpha / 2}([0,1] ; Z)}+\|u\|_{C^{\alpha / 2}([0,1] ; D(A))}+\left\|u^{\prime}\right\|_{B\left([0,1] ; D_{A}(\alpha / 2, \infty)\right)} & \\
& \leqslant C\left(\left\|u_{0}\right\|_{D(A)}+\left\|A u_{0}+f(0)\right\|_{D_{A}(\alpha / 2, \infty)}+\|f\|_{C^{\alpha / 2}([0,1] ; Z)}\right) .
\end{aligned}
$$

Proof. It is well known (see e.g. [10, Theorem 4.3.8]) that, under our assumptions, formula (4.38) defines the unique classical solution of the equation $u^{\prime}=A u+f$. Moreover, if $u_{0} \in \overline{D(A)}$ and $A u_{0}+f(0) \in D_{A}(\alpha / 2, \infty)$, then $u \in C^{\alpha / 2}([0, T] ; D(A)) \cap C^{1+\alpha / 2}([0, T] ; Z)$ and $u^{\prime} \in$ $B\left([0, T] ; D_{A}(\alpha / 2, \infty)\right)$ for any $T>0$, and 44.40 is satisfied. Hence, we can limit ourselves to dealing with the asymptotic behaviour of $u$, simply by checking 44.39 . For this purpose we observe that, $e^{t A}$ being a semigroup of negative type, there exist positive constants $\omega$ and $M_{k}(k \in \mathbb{N})$ such that

$$
t^{k}\left\|A^{k} e^{t A}\right\|_{L(Z)} \leqslant M_{k} e^{-\omega t}, \quad \forall t>0, k \in \mathbb{N} .
$$

Taking 4.41 into account, it is now easy to show that the functions $u_{1}(t)=e^{t A} u_{0}$ and $A u_{1}$ decay exponentially at infinity. In particular, there exists a positive constant $C$ such that 


$$
\begin{array}{r}
\sup _{t \geqslant 1}(1+t)\left(\left\|u_{1}(t)\right\|_{D(A)}+\left\|u_{1}^{\prime}(t)\right\|_{D_{A}(\alpha / 2, \infty)}\right)+\sup _{1 \leqslant s<t}(1+s) \frac{\left\|u_{1}^{\prime}(t)-u_{1}^{\prime}(s)\right\|_{Z}}{(t-s)^{\alpha / 2}} \\
\leqslant C\left\|u_{0}\right\|_{Z} .
\end{array}
$$

Let us now consider the integral term $u_{2}(t)=\int_{0}^{t} e^{(t-s) A} f(s) \mathrm{d} s$. As is easily seen,

$$
\begin{aligned}
\left\|u_{2}(t)\right\|_{Z} & \leqslant \sup _{r \geqslant 0}(1+r)\|f(r)\|_{Z}\left(\int_{0}^{t / 2}(1+s)^{-1} e^{-\omega(t-s)} \mathrm{d} s+\int_{t / 2}^{t}(1+s)^{-1} e^{-\omega(t-s)} \mathrm{d} s\right) \\
& \leqslant \sup _{r \geqslant 0}(1+r)\|f(r)\|_{Z}\left(e^{-\omega t / 2} \int_{0}^{t / 2}(1+s)^{-1} \mathrm{~d} s+(1+t / 2)^{-1} \omega^{-1}\right) \\
& \leqslant C(1+t)^{-1} \sup _{r \geqslant 0}(1+r)\|f(r)\|_{Z}, \quad \forall t>0 .
\end{aligned}
$$

Similarly, since

$$
u_{2}^{\prime}(t)=\int_{0}^{t} A e^{(t-s) A}(f(s)-f(t)) \mathrm{d} s+e^{t A} f(t), \quad \forall t>0,
$$

we easily deduce that

$$
\begin{aligned}
\left\|u_{2}^{\prime}(t)\right\|_{Z} \leqslant & M_{1} \sup _{0 \leqslant \sigma<\tau}(1+\sigma) \frac{\|f(\tau)-f(\sigma)\|_{Z}}{(\tau-\sigma)^{\alpha / 2}} \int_{0}^{t}(1+s)^{-1}(t-s)^{\alpha / 2-1} e^{-\omega(t-s)} \mathrm{d} s \\
& +M_{0} e^{-\omega t} \sup _{t \geqslant 0}\|f(t)\|_{Z} \\
\leqslant & M_{1}\left((t / 2)^{\alpha / 2} e^{-\omega t / 2}+(1+t / 2)^{-1} \int_{0}^{+\infty} s^{\alpha / 2-1} e^{-\omega s} \mathrm{~d} s\right)[[f]]_{3} \\
& +M_{0} e^{-\omega t} \sup _{t \geqslant 0}\|f(t)\|_{Z} \\
\leqslant & C(1+t)^{-1}[[f]]_{3}, \quad \forall t>0 .
\end{aligned}
$$

Moreover, for any $0 \leqslant s<t$,

$$
\begin{aligned}
u_{2}^{\prime}(t)-u_{2}^{\prime}(s)= & \int_{0}^{s} A\left(e^{(t-\sigma) A}-e^{(s-\sigma) A}\right)(f(\sigma)-f(s)) \mathrm{d} \sigma+\left(e^{t A}-e^{s A}\right) f(s) \\
& +e^{(t-s) A}(f(t)-f(s))+\int_{s}^{t} A e^{(t-\sigma) A}(f(\sigma)-f(t)) \mathrm{d} \sigma .
\end{aligned}
$$

Let us now consider the first term in 4.45 and observe that

$$
\begin{aligned}
& \left\|\int_{0}^{s} A\left(e^{(t-\sigma) A}-e^{(s-\sigma) A}\right)(f(\sigma)-f(s)) \mathrm{d} \sigma\right\|_{Z}=\left\|\int_{0}^{s} \mathrm{~d} \sigma \int_{s-\sigma}^{t-\sigma} A^{2} e^{\tau A}(f(\sigma)-f(s)) \mathrm{d} \tau\right\|_{Z} \\
& \leqslant M_{2} \sup _{0 \leqslant r_{1}<r_{2}}\left(1+r_{1}\right) \frac{\left\|f\left(r_{2}\right)-f\left(r_{1}\right)\right\|_{Z}}{\left(r_{2}-r_{1}\right)^{\alpha / 2}} \int_{0}^{s}(1+\sigma)^{-1} \mathrm{~d} \sigma \int_{s-\sigma}^{t-\sigma} \tau^{\alpha / 2-2} e^{-\omega \tau} \mathrm{d} \tau \\
& \leqslant M_{2}[[f]]_{3}\left(e^{-\omega s / 2} \int_{0}^{s / 2} \mathrm{~d} \sigma \int_{s-\sigma}^{t-\sigma} \tau^{\alpha / 2-2} \mathrm{~d} \tau+(1+s / 2)^{-1} \int_{s / 2}^{s} \mathrm{~d} \sigma \int_{s-\sigma}^{t-\sigma} \tau^{\alpha / 2-2} e^{-\omega \tau} \mathrm{d} \tau\right) \\
& \leqslant C[[f]]_{3} .
\end{aligned}
$$


All the other terms are easily estimated and have estimates similar to (4.46). Hence, from (4.42) and (4.46) we deduce that there exists a positive constant $C$ such that

$$
\sup _{1 \leqslant s<t}(1+s) \frac{\left\|u^{\prime}(t)-u^{\prime}(s)\right\|_{Z}}{(t-s)^{\alpha / 2}} \leqslant C\left(\left\|u_{0}\right\|_{Z}+[[f]]_{3}\right) .
$$

Finally, let us estimate the asymptotic behaviour of $\left\|u^{\prime}(t)\right\|_{D_{A}(\alpha / 2, \infty)}$. For this purpose, fix $\xi \in$ $(0,1), t \geqslant 1$, and observe that

$$
\begin{aligned}
& \xi^{1-\alpha / 2}\left\|A e^{\xi A} u_{2}^{\prime}(t)\right\|_{Z} \\
& \leqslant \xi^{1-\alpha / 2}\left\|\int_{0}^{t} A^{2} e^{(t+\xi-s) A}(f(s)-f(t)) \mathrm{d} s\right\|_{Z}+\xi^{1-\alpha / 2}\left\|A e^{(t+\xi) A} f(t)\right\|_{Z} \\
& \leqslant M_{2} \xi^{1-\alpha / 2} \sup _{0 \leqslant \sigma<\tau}(1+\sigma) \frac{\|f(\tau)-f(\sigma)\|_{Z}}{(\tau-\sigma)^{\alpha / 2}} \int_{0}^{t} \frac{e^{-\omega(t+\xi-s)}(t-s)^{\alpha / 2}}{(t+\xi-s)^{2}(1+s)} \mathrm{d} s \\
& \quad+M_{1} \xi^{1-\alpha / 2} \frac{e^{-\omega(\xi+t)}}{t+\xi}\|f(t)\|_{Z} \\
& \leqslant M_{2} \xi^{1-\alpha / 2}[[f]]_{3}\left(\frac{t^{\alpha / 2} e^{-\omega(\xi+t / 2)}}{(\xi+t / 2)^{2}} \int_{0}^{t / 2} \frac{\mathrm{d} s}{1+s}+\frac{1}{1+t / 2} \int_{t / 2}^{t} \frac{\mathrm{d} s}{(t+\xi-s)^{2-\alpha / 2}}\right) \\
& \quad+M_{1} \xi^{1-\alpha / 2} \frac{e^{-\omega(\xi+t)}}{t+\xi}\|f(t)\|_{Z} .
\end{aligned}
$$

Taking the supremum over all $\xi \in(0,1)$ gives

$$
\sup _{\xi \in(0,1)} \xi^{1-\alpha / 2}\left\|A e^{\xi A} u_{2}^{\prime}(t)\right\|_{Z} \leqslant C(1+t)^{-1}[[f]]_{3} .
$$

Since $\left\|u_{2}^{\prime}(t)\right\|_{D_{A}(\alpha / 2, \infty)}=\left\|u_{2}(t)\right\|_{Z}+\sup _{\xi \in(0,1)} \xi^{1-\alpha / 2}\left\|A e^{\xi A} u_{2}^{\prime}(t)\right\|_{Z}$, we get

$$
\left\|u_{2}^{\prime}(t)\right\|_{D_{A}(\alpha / 2, \infty)} \leqslant C(1+t)^{-1}[[f]]_{3}
$$

The assertion now follows from (4.42)-4.44, (4.47) and 4.49) and our assumptions on $f$, since $u^{\prime}=A u+f$.

Next we deal with the function

$$
\mathbf{u}(t, \cdot)=L \int_{0}^{t} e^{(t-s) L} \mathcal{N} \psi(s, \cdot) \mathrm{d} s,
$$

where $\mathcal{N}$ is the lifting operator defined in Lemma 4.5

LEMMA 4.9 Suppose that $\psi \in C^{(1+\alpha) / 2,1+\alpha}([0,+\infty) \times \mathbb{R})(\alpha \in(0,1))$ is such that

$$
[[\psi]]_{4}=\sup _{t \geqslant 0}(1+t)\|\psi(t, \cdot)\|_{C_{b}^{1+\alpha}(\mathbb{R})}+\sup _{0 \leqslant s<t}(1+s) \frac{\|\psi(t, \cdot)-\psi(s, \cdot)\|_{C_{b}(\mathbb{R})}}{(t-s)^{(1+\alpha) / 2}}<+\infty .
$$


Then the function $\mathbf{u}$ given by 4.50$]$ belongs to $\mathcal{X}_{1+\alpha / 2,2+\alpha}(1, \infty)$. Moreover, there exists a positive constant $C$, independent of $\psi$, such that

$$
\begin{aligned}
& \sup _{t \geqslant 1}(1+t)\left(\|\mathbf{u}(t, \cdot)\|_{X_{2+\alpha}}+\left\|D_{t} \mathbf{u}(t, \cdot)\right\|_{X_{\alpha}}\right)+\sup _{1 \leqslant s<t}(1+s) \frac{\|\mathbf{u}(t, \cdot)-\mathbf{u}(s, \cdot)\|_{X_{2}}}{(t-s)^{\alpha / 2}} \\
& \quad+\sup _{1 \leqslant s<t}(1+s)\left(\frac{\|\mathbf{u}(t, \cdot)-\mathbf{u}(s, \cdot)\|_{X_{1}}}{(t-s)^{(1+\alpha) / 2}}+\frac{\left\|D_{t} \mathbf{u}(t, \cdot)-D_{t} \mathbf{u}(s, \cdot)\right\|_{X_{0}}}{(t-s)^{\alpha / 2}}\right) \\
& \leqslant C[[\psi]]_{4} .
\end{aligned}
$$

Proof. Throughout the proof, we denote by $C_{j}(j \in \mathbb{N})$ positive constants, independent of the data and $t$.

By assumptions $\psi \in C^{(1+\alpha) / 2,1+\alpha}([0,+\infty) \times \mathbb{R})$. Hence, $\mathcal{N} \psi \in B\left([0,+\infty) ; X_{2+\alpha}\right)$ (cf. Lemma 4.5. Moreover, there exists a positive constant $C_{1}$ such that

$$
\sup _{t \geqslant 0}(1+t)\|\mathcal{N} \psi(t, \cdot)\|_{X_{2+\alpha}} \leqslant C_{1} \sup _{t \geqslant 0}(1+t)\|\psi(t, \cdot)\|_{C_{b}^{1+\alpha}(\mathbb{R})} .
$$

From Lemma 4.5 we deduce that

$$
\begin{aligned}
(1+s)\|\mathcal{N} \psi(t, \cdot)-\mathcal{N} \psi(s, \cdot)\|_{X_{1}} & \leqslant C_{2}(1+s)\|\psi(t, \cdot)-\psi(s, \cdot)\|_{C_{b}(\mathbb{R})} \\
& \leqslant C_{2}[[\psi]]_{4}(t-s)^{(1+\alpha) / 2},
\end{aligned}
$$

for any $0 \leqslant s<t$ and some positive constant $C_{2}$. Hence, $\mathcal{N} \psi$ belongs to $C^{(1+\alpha) / 2}\left([0,+\infty) ; X_{1}\right)$.

Since $\left\{\mathbf{f} \in(I-P)\left(X_{1}\right): B_{0} \mathbf{f}=0\right.$ at $\left.x=0\right\}$ is continuously embedded in the space $Z:=$ $(I-P)\left(D_{L}(1 / 2, \infty)\right)$ endowed with the norm of $D_{L}(1 / 2, \infty)$ (cf. Theorem 4.1) and $\mathcal{N} \psi(t, \cdot) \in$ $(I-P)\left(X_{0}\right)$ for any $t \geqslant 0$, it follows that $\mathcal{N} \psi \in C^{(1+\alpha) / 2}([0,+\infty) ; Z)$. Moreover, from 4.52 and (4.53) we deduce that there exists a positive constant $C_{3}$ such that

$$
\sup _{t \geqslant 0}\|\mathcal{N} \psi(t, \cdot)\|_{D_{L}(1 / 2, \infty)}+\sup _{0 \leqslant s<t}(1+s) \frac{\|\mathcal{N} \psi(t, \cdot)-\mathcal{N} \psi(s, \cdot)\|_{D_{L}(1 / 2, \infty)}}{(t-s)^{(1+\alpha) / 2}} \leqslant C_{3}[[\psi]]_{4} .
$$

Observe that the function $\mathcal{N} \psi$ satisfies all the assumptions of Lemma 4.8 (see Lemma 4.5), and the restriction of $e^{t L}$ to $Z$ defines an analytic semigroup of negative type (see Theorem 4.3 whose generator is the part of $L$ in $Z$. Hence, Lemma 4.8 and 4.54 imply that the function $\mathbf{w}(t, \cdot)=$ $\int_{0}^{t} e^{(t-s) L} \mathcal{N} \psi(s, \cdot) \mathrm{d} s$ belongs to $Z$ for any $t \geqslant 0$, and satisfies the following estimate:

$$
\sup _{t \geqslant 1}(1+t)\left(\|L \mathbf{w}(t, \cdot)\|_{D_{L}(1 / 2, \infty)}+\left\|D_{t} \mathbf{w}(t, \cdot)\right\|_{D_{L}(1+\alpha / 2, \infty)}\right) \leqslant C_{4}[[\psi]]_{4} .
$$

Let us prove that $L \mathbf{w} \in C_{b}^{1+\alpha / 2}\left([1,+\infty) ; X_{0}\right)$. For this purpose, we begin by observing that $L \mathbf{w}$ is differentiable with respect to time and $D_{t}(L \mathbf{w})(t, \cdot)=L D_{t} \mathbf{w}(t, \cdot)$ for any $t \geqslant 0$. Indeed, since $B\left([0,+\infty) ; D_{L}(1+\alpha / 2, \infty)\right) \subset C([0,+\infty) ; D(L))$, we have

$$
L \mathbf{w}(t, \cdot)-L \mathbf{w}(s, \cdot)=\int_{s}^{t} L D_{t} \mathbf{w}(\sigma, \cdot) \mathrm{d} \sigma, \quad \forall t, s \geqslant 0 .
$$

Consequently, $L \mathbf{w}$ is differentiable with respect to $t$ in $[0,+\infty)$ and $D_{t} L \mathbf{w}=L D_{t} \mathbf{w}$. Hence, we can write

$$
L D_{t} \mathbf{w}(t, \cdot)=\int_{0}^{t} L^{2} e^{(t-s) L}(\mathcal{N} \psi(s, \cdot)-\mathcal{N} \psi(t, \cdot)) \mathrm{d} s+L e^{t L} \mathcal{N} \psi(t, \cdot)
$$


We fix now, for the rest of the proof, a positive constant $\omega \in(0,1 / 4)$. Then, by $(4.3)$, we know that there exist positive constants $M_{k}(k \in \mathbb{N})$ such that

$$
\sup _{t>0} t^{k-1 / 2}\left\|L^{k} e^{t L}(I-P)\right\|_{L\left(D_{L}(1 / 2, \infty), X_{0}\right)} \leqslant M_{k} e^{-\omega t} .
$$

From 4.56 it immediately follows that

$$
\begin{aligned}
\left\|D_{t} L \mathbf{w}(t, \cdot)\right\|_{X_{0}} \leqslant & \int_{0}^{t}\left\|L^{2} e^{(t-s) L}(\mathcal{N} \psi(s, \cdot)-\mathcal{N} \psi(t, \cdot))\right\|_{X_{0}} \mathrm{~d} s+\left\|L e^{t L} \mathcal{N} \psi(t, \cdot)\right\|_{X_{0}} \\
\leqslant & M_{2} \sup _{0 \leqslant \sigma<\tau}(1+\sigma) \frac{\|\psi(\tau, \cdot)-\psi(\sigma, \cdot)\|_{C_{b}(\mathbb{R})}}{(\tau-\sigma)^{(1+\alpha) / 2}} \int_{0}^{t}(1+s)^{-1}(t-s)^{-1+\alpha / 2} e^{-\omega(t-s)} \mathrm{d} s \\
& +2 M_{1} t^{-3 / 2} e^{-\omega t}\|\psi(t, \cdot)\|_{C_{b}(\mathbb{R})} \\
\leqslant & M_{2}[[\psi]]_{4}\left(e^{-\omega t / 2} \int_{0}^{t} s^{\alpha / 2-1} \mathrm{~d} s+(t / 2)^{-1} \int_{0}^{t} s^{\alpha / 2-1} e^{-\omega s} \mathrm{~d} s\right) \\
& +2 M_{1} t^{-1}\|\psi(t, \cdot)\|_{C_{b}(\mathbb{R})} \\
\leqslant & C_{5} t^{-1}[[\psi]]_{4},
\end{aligned}
$$

for any $t \geqslant 1$. Moreover,

$$
\begin{aligned}
\left\|D_{t} L \mathbf{w}(t, \cdot)-D_{t} L \mathbf{w}(s, \cdot)\right\|_{X_{0}} & \left\|\int_{0}^{s} L^{2}\left(e^{(t-\sigma) L}-e^{(s-\sigma) L}\right)(\mathcal{N} \psi(\sigma, \cdot)-\mathcal{N} \psi(s, \cdot)) \mathrm{d} \sigma\right\|_{X_{0}}+\left\|L\left(e^{t L}-e^{s L}\right) \mathcal{N} \psi(s, \cdot)\right\|_{X_{0}} \\
\leqslant & +\left\|\int_{s}^{t} L^{2} e^{(t-\sigma) L}(\mathcal{N} \psi(\sigma, \cdot)-\mathcal{N} \psi(t, \cdot)) \mathrm{d} \sigma\right\|_{X_{0}}+\left\|L e^{(t-s) L}(\mathcal{N} \psi(t, \cdot)-\mathcal{N} \psi(s, \cdot))\right\|_{X_{0}} \\
\leqslant & C_{6}[[\psi]]_{4}\left(\int_{0}^{s} \frac{(s-\sigma)^{(1+\alpha) / 2}}{1+\sigma} \mathrm{d} \sigma \int_{s-\sigma}^{t-\sigma} \frac{e^{-\omega \tau}}{\tau^{5 / 2}} \mathrm{~d} \tau+\int_{s}^{t} \sigma^{-3 / 2} e^{-\omega \sigma} \mathrm{d} \sigma\right. \\
& \left.+\int_{s}^{t} \frac{e^{-\omega(t-\sigma)}}{(t-\sigma)^{1-\alpha / 2}} \frac{1}{1+\sigma} \mathrm{d} \sigma+\frac{1}{1+s}\left\|L e^{(t-s) L}\right\|_{L\left(D_{L}(1 / 2, \infty), X_{0}\right)}(t-s)^{(1+\alpha) / 2}\right) \\
\leqslant & C_{7}[[\psi]]_{4}\left(\int_{0}^{s} \frac{e^{-\omega(s-\sigma)}}{1+\sigma} \mathrm{d} \sigma \int_{s-\sigma}^{t-\sigma} \frac{1}{\tau^{2-\alpha / 2}} \mathrm{~d} \tau+\frac{1}{1+s} \int_{s}^{t} \frac{e^{-\omega(t-\sigma)}}{(t-\sigma)^{1-\alpha / 2}} \mathrm{~d} \sigma\right. \\
& +\left(\frac{e^{-\omega s}}{\left.\left.s^{(1+\alpha) / 2}+\frac{1}{s+1}\right)(t-s)^{\alpha / 2}\right)}\right. \\
\leqslant & C_{8}[[\psi]]_{4}\left(e^{-\omega s / 2} \int_{0}^{s / 2} \mathrm{~d} \sigma \int_{s-\sigma}^{t-\sigma} \frac{1}{\tau^{2-\alpha / 2}} \mathrm{~d} \tau+\frac{1}{1+s} \int_{s / 2}^{s} \mathrm{~d} \sigma \int_{s-\sigma}^{t-\sigma} \frac{1}{\tau^{2-\alpha / 2}} \mathrm{~d} \tau\right. \\
& \left.+e^{-\omega s}(t-s)^{\alpha / 2}+\frac{1}{1+s}(t-s)^{\alpha / 2}\right) \\
\leqslant & C_{9}[[\psi]]_{4}(1+s)^{-1}(t-s)^{\alpha / 2},
\end{aligned}
$$

for any $0 \leqslant s<t$. Observe that $\mathbf{u}=L \mathbf{w}$; then (4.55), (4.57) and (4.58) imply that $D_{t} \mathbf{u} \in$ $B\left([0,+\infty) ; X_{\alpha}\right) \cap C^{\alpha / 2}\left([0,+\infty) ; X_{0}\right)$ and

$$
(1+t) \sup _{t \geqslant 1}\left\|D_{t} \mathbf{u}(t, \cdot)\right\|_{X_{\alpha}}+\sup _{1 \leqslant s<t}(1+s) \frac{\left\|D_{t} \mathbf{u}(t, \cdot)-D_{t} \mathbf{u}(s, \cdot)\right\|_{X_{0}}}{(t-s)^{\alpha / 2}} \leqslant C_{10}[[\psi]]_{4}, \quad \forall t \geqslant 1 .
$$


Since $\mathbf{u}+\mathcal{N} \psi=D_{t} \mathbf{w} \in B\left([0,+\infty) ; D_{L}(1+\alpha / 2, \infty)\right)($ see 4.55$\left.]\right)$ and $\mathcal{N} \psi \in B\left([0,+\infty) ; X_{2+\alpha}\right)$ (see (4.52)), we easily deduce that $\mathbf{u} \in B\left([0,+\infty), X_{2+\alpha}\right)$ and there exists a positive constant $C_{11}$ such that

$$
\sup _{t \geqslant 1}(1+t)\|\mathbf{u}(t, \cdot)\|_{X_{2+\alpha}} \leqslant C_{11}[[\psi]]_{4} .
$$

Now, taking (4.55), 4.59), 4.60) into account and applying Lemma 3.4 to the function $\mathbf{v}$ defined by $\mathbf{v}(t, \cdot)=\mathbf{u}(t+1, \cdot)$ for any $t \geqslant 0$, we easily get 4.51 . The proof is now complete.

We can now prove Theorem 4.7

Proof of Theorem 4.7. The statement follows from Theorem 4.6 and Lemmas 4.8, 4.9 and A.3. Indeed, our assumptions on the triplet $\left(\mathbf{u}_{0}, \mathbf{g}, \psi\right)$, the compatibility conditions in (4.35) and Theorem 4.6 imply that problem $4.17 \mathrm{i}$ ) admits a unique solution $\mathbf{w}$ which belongs to $\mathcal{X}_{1+\alpha / 2,2+\alpha}(0, T)$ for any $T>0$ and satisfies 4.32 .

Let us recall that the solution to problem (4.17)i) can be split as $\mathbf{w}=\mathbf{w}_{1}+\mathbf{w}_{2}$, where

$$
\begin{aligned}
& \mathbf{w}_{1}(t, \cdot):=e^{t L} \mathbf{u}_{0}+\int_{0}^{t} e^{(t-s) L}\left(\mathbf{g}(s, \cdot)+\psi(s, \cdot) \mathbf{U}_{0}+\mathcal{L N} \psi(s, \cdot)\right) \mathrm{d} s, \\
& \mathbf{w}_{2}(t, \cdot):=-L \int_{0}^{t} e^{(t-s) L} \mathcal{N} \psi(s, \cdot) \mathrm{d} s, \quad \forall t \geqslant 0 .
\end{aligned}
$$

Throughout the rest of the proof, we denote by $C_{j}(j \in \mathbb{N})$ positive constants, independent of the data and $t$.

Due to Lemma 4.5.iii) and 4.52, the function $t \mapsto \mathbf{g}(t, \cdot)+\psi(t, \cdot) \mathbf{U}_{0}+\mathcal{L N} \psi(t, \cdot)$ belongs to $(I-P)\left(X_{0}\right)$ for any $t \geqslant 0$. Moreover, it satisfies condition 4.37). To check it, we begin by observing that

$$
\sup _{0 \leqslant s<t}(1+s) \frac{\|\psi(t, \cdot)-\psi(s, \cdot)\|_{C_{b}(\mathbb{R})}}{(t-s)^{\alpha / 2}}<2[[\psi]]_{2},
$$

which follows easily by observing that

$$
\|\psi(t, \cdot)-\psi(s, \cdot)\|_{C_{b}(\mathbb{R})} \leqslant(1+s)^{-1}[[\psi]]_{2}(t-s)^{(1+\alpha) / 2} \leqslant(1+s)^{-1}[[\psi]]_{2}(t-s)^{\alpha / 2}
$$

if $0 \leqslant s<t$ and $t \leqslant s+1$, while

$$
\|\psi(t, \cdot)-\psi(s, \cdot)\|_{C_{b}(\mathbb{R})} \leqslant\left(\|\psi(t, \cdot)\|_{C_{b}(\mathbb{R})}+\|\psi(s, \cdot)\|_{C_{b}(\mathbb{R})}\right)(t-s)^{\alpha / 2} \leqslant 2(1+s)^{-1}[[\psi]]_{2}(t-s)^{\alpha / 2}
$$

if $0 \leqslant s<t$ and $t>s+1$.

Now, taking 4.34, 4.61 and Lemma 4.5 into account, we easily deduce that

$$
\begin{aligned}
\|\mathcal{N} \psi(t, \cdot)-\mathcal{N} \psi(s, \cdot)\|_{X_{2}} & \leqslant C_{1}\|\psi(t, \cdot)-\psi(s, \cdot)\|_{C_{b}^{1}(\mathbb{R})} \\
& \leqslant 2 C_{1}[[\psi]]_{2}(1+s)^{-1}(t-s)^{\alpha / 2}, \quad \forall 0 \leqslant s<t .
\end{aligned}
$$

Hence, from 4.52, (4.61) and 4.62) we get

$$
\left[\left[\psi \mathbf{U}_{0}+\mathcal{L N} \psi\right]\right]_{3} \leqslant C_{2}[[\psi]]_{2} .
$$


Applying Lemma 4.8 with $Z=(I-P)\left(X_{0}\right), A=L_{\mid(I-P)}, f=\mathbf{g}+\psi \mathbf{U}_{0}+\mathcal{L N} \psi, u_{0}=\mathbf{w}_{0}$, and taking into account the characterization of the interpolation space $D_{L}(\alpha / 2, \infty)$ given in Theorem 4.1, and 4.63, we get

$$
\begin{array}{r}
\sup _{t \geqslant 1}(1+t)\left(\left\|\mathbf{w}_{1}(t, \cdot)\right\|_{D(L)}+\left\|D_{t} \mathbf{w}_{1}(t, \cdot)\right\|_{X_{\alpha}}\right)+\sup _{1 \leqslant s<t}(1+s) \frac{\left\|D_{t} \mathbf{w}_{1}(t, \cdot)-D_{t} \mathbf{w}_{1}(s, \cdot)\right\|_{X_{0}}}{(t-s)^{\alpha / 2}} \\
\leqslant C_{3}\left(\left\|\mathbf{w}_{0}\right\|_{X_{0}}+[[\mathbf{g}]]_{1}+[[\psi]]_{2}\right) .
\end{array}
$$

Since $D_{t} \mathbf{w}_{1}=L \mathbf{w}_{1}+\mathbf{g}+\psi \mathbf{U}_{0}+\mathcal{L} \mathcal{N} \psi$ and $\mathbf{g}+\psi \mathbf{U}_{0}+\mathcal{L} \mathcal{N} \psi \in B\left([0,+\infty) ; X_{\alpha}\right)$ with

$$
\sup _{t \geqslant 0}(1+t)\left\|\mathbf{g}(t, \cdot)+\psi(t, \cdot) \mathbf{U}_{0}+\mathcal{L N} \psi(t, \cdot)\right\|_{X_{\alpha}} \leqslant C_{4}\left([[\mathbf{g}]]_{1}+[[\psi]]_{2}\right)
$$

we easily deduce that

$$
\sup _{t \geqslant 1}(1+t)\left\|L \mathbf{w}_{1}(t, \cdot)\right\|_{X_{\alpha}} \leqslant C_{5}\left(\left\|\mathbf{w}_{0}\right\|_{X_{0}}+[[\mathbf{g}]]_{1}+[[\psi]]_{2}\right) .
$$

From the Schauder estimate in [1] and from (4.64) and 4.65] it easily follows that $\mathbf{w}_{1}$ belongs to $B\left([0,+\infty) ; X_{2+\alpha}\right)$ and

$$
\sup _{t \geqslant 1}(1+t)\left\|\mathbf{w}_{1}(t, \cdot)\right\|_{X_{2+\alpha}} \leqslant C_{6}\left(\left\|\mathbf{w}_{0}\right\|_{X_{0}}+[[\mathbf{g}]]_{1}+[[\psi]]_{2}\right) .
$$

Now, applying Lemma 3.4 to the function $\mathbf{u}(t, \cdot)=\mathbf{w}(t+1, \cdot)$ and taking 4.64) and 4.66 into account, we get

$$
\begin{array}{r}
\sup _{1 \leqslant s<t}(1+s) \frac{\left\|\mathbf{w}_{1}(t, \cdot)-\mathbf{w}_{1}(s, \cdot)\right\|_{X_{2}}}{(t-s)^{\alpha / 2}}+\sup _{1 \leqslant s<t}(1+s) \frac{\left\|\mathbf{w}_{1}(t, \cdot)-\mathbf{w}_{1}(s, \cdot)\right\|_{X_{1}}}{(t-s)^{(1+\alpha) / 2}} \\
\leqslant C_{7}\left(\left\|\mathbf{w}_{0}\right\|_{X_{0}}+[[\mathbf{g}]]_{1}+[[\psi]]_{2}\right) .
\end{array}
$$

As far as the term $\mathbf{w}_{2}$ is concerned, we observe that the assumptions of Lemma 4.9 are satisfied by $\psi$. Hence, from 4.32) (with $T=1$ ), 4.51) (applied to the function $\mathbf{w}_{2}$ ), 4.64), 4.66), 4.67) and Lemma A.3. we deduce that $\mathbf{w}$ belongs to $\mathcal{X}_{\mathbf{w}}$ and satisfies 4.36.

\subsection{Stability results}

This subsection is devoted to proving that the null solution to problem 2.15) is stable with respect to smooth and sufficiently small perturbations. Theorem 4.7 provides a useful tool to prove our stability result. As already pointed out, we can limit ourselves to dealing with problems (4.16) and 4.16 ii) where $\widetilde{\mathcal{H}}, \mathcal{K}$ and $\mathcal{G}$ are given, respectively, by $4.12,4.13-\sqrt{4.15}$ and 2.14 .

We solve system 4.16 by a fixed point argument. Indeed, any sufficiently smooth solution to system 4.16) is a fixed point of the operator $\Gamma(q, \mathbf{w})=\left(\Gamma_{1}(q, \mathbf{w}), \Gamma_{2}(q, \mathbf{w})\right)$ defined by

$$
\begin{aligned}
\Gamma_{1}(q, \mathbf{w})(t, \cdot)= & T(t)\left(\exp \left(M\left(\mathbf{u}_{0}\right) / 2\right)-1\right)+\int_{0}^{t} T(t-s) \tilde{\mathcal{H}}(q(s, \cdot), \mathbf{w}(s, \cdot)) \mathrm{d} s, \quad t \geqslant 0 \\
\Gamma_{2}(q, \mathbf{w})(t, \cdot)= & e^{t L}(I-P) \mathbf{u}_{0} \\
& +\int_{0}^{t} e^{(t-s) L}(\mathcal{K}(q(s, \cdot), \mathbf{w}(s, \cdot))+\mathcal{L} \mathcal{N} \widetilde{g}(q(s, \cdot), \mathbf{w}(s, \cdot)) \mathrm{d} s \\
& -L \int_{0}^{t} e^{(t-s) L} \mathcal{N} \widetilde{g}(q(s, \cdot), \mathbf{w}(s, \cdot)) \mathrm{d} s, \quad t \geqslant 0 .
\end{aligned}
$$


Let us introduce the Banach space where we are going to solve the fixed point equation for $(q, \mathbf{w})$. We denote by $\mathcal{X}_{q, \mathbf{w}}$ the Banach space of all pairs $(q, \mathbf{w}) \in \mathcal{X}_{q} \times \mathcal{X}_{\mathbf{w}}$ such that $\mathbf{w}(t, \cdot) \in(I-P)\left(X_{0}\right)$ for any $t \geqslant 0$ (see Definition 3.3p, endowed with the norm $\|(q, \mathbf{w})\|_{\mathcal{X}_{q, \mathbf{w}}}=\|q\|_{\mathcal{X}_{q}}+\|\mathbf{w}\|_{\mathcal{X}_{\mathbf{w}}}$, and we denote by $B(\mathbf{0}, \rho)$ its closed ball with centre at $(0, \mathbf{0})$ and radius $\rho$.

The main result of this subsection is the following theorem.

THEOREM 4.10 Suppose that $q_{0} \in C_{b}^{2+\alpha}(\mathbb{R})$ and $\mathbf{w}_{0} \in(I-P)\left(X_{2+\alpha}\right)$ satisfy the compatibility conditions

$$
\mathcal{B} \mathbf{w}_{0}=\mathcal{G}\left(\mathbf{w}_{0}+2 \log \left(q_{0}+1\right) \mathbf{U}_{0}\right), \quad B_{0} \mathcal{L}\left(\mathbf{w}_{0}+\mathcal{K}\left(q_{0}, \mathbf{w}_{0}\right)\right)=0 .
$$

Then there are positive constants $\rho_{0}$ and $\rho$ such that if

$$
\left\|q_{0}\right\|_{C_{b}^{2+\alpha}(\mathbb{R})}+\left\|\mathbf{w}_{0}\right\|_{X_{2+\alpha}} \leqslant \rho_{0}
$$

then problem 4.16 admits a unique solution $(q, \mathbf{w}) \in B(\mathbf{0}, \rho)$ satisfying $\left(q(0, \cdot), \mathbf{w}_{0}(0, \cdot)\right)=$ $\left(q_{0}, \mathbf{w}_{0}\right)$. Moreover, there exists a positive constant $C$, independent of $(q, \mathbf{w})$, such that

$$
\begin{array}{r}
\|(q, \mathbf{w})\|_{\mathcal{X}_{q, \mathbf{w}}}+\sup _{0 \leqslant s<t}(1+s)\left(\frac{\left\|q_{y}(t, \cdot)-q_{t}(s, \cdot)\right\|_{C_{b}(\mathbb{R})}}{(t-s)^{(1+\alpha) / 2}}+\frac{\|\mathbf{w}(t, \cdot)-\mathbf{w}(s, \cdot)\|_{X_{1}}}{(t-s)^{(1+\alpha) / 2}}\right) \\
\leqslant C\left(\left\|q_{0}\right\|_{C_{b}^{2+\alpha}(\mathbb{R})}+\left\|\mathbf{w}_{0}\right\|_{X_{2+\alpha}}\right) .
\end{array}
$$

Proof. Let us prove that the operator $\Gamma$ defined by (4.68) is a contraction mapping in the space

$$
B\left(\mathbf{0},\left(q_{0}, \mathbf{w}_{0}\right), \rho\right):=\left\{(q, \mathbf{w}) \in B(\mathbf{0}, \rho):(q(0, \cdot), \mathbf{w}(0, \cdot))=\left(q_{0}, \mathbf{w}_{0}\right)\right\},
$$

provided $\rho_{0}$ and $\rho$ are sufficiently small. For this purpose let us estimate the functions $\widetilde{g}, \tilde{\mathcal{H}}$ and $\mathcal{K}$. We begin with $\widetilde{g}$ (see (4.14)). Observe that, taking Lemma 3.4 into account, one can easily show that there exists a positive constant $C$, independent of $q$, such that

$$
\sup _{s \geqslant 0}(1+s)^{j / 2}\left(\left\|D_{y}^{j} q(s, \cdot)\right\|_{C_{b}^{\alpha}(\mathbb{R})}+\left\|D_{y}^{j} q\right\|_{C^{(i+\alpha) / 2}\left([s,+\infty) ; C_{b}(\mathbb{R})\right)}\right) \leqslant C\|q\|_{\mathcal{X}_{q}}, \quad \forall 0 \leqslant i, j \leqslant 1 .
$$

Since $\widetilde{g}$ is a product of functions belonging to $C^{(1+\alpha) / 2,1+\alpha}([0,+\infty) \times \mathbb{R})$, we easily deduce that, if $\rho$ is taken sufficiently small so that both $1+q$ and $1-v(0)+v_{x}(0)$ never vanish for $(q, \mathbf{w}) \in B(\mathbf{0}, \rho)$ (e.g. $\rho \leqslant 1 / 2)$, then $\widetilde{g}(q, \mathbf{w}) \in C^{(1+\alpha) / 2,1+\alpha}([0,+\infty) \times \mathbb{R})$. Moreover, long but straightforward computations and the fact that $\widetilde{g}$ is quadratic near $\mathbf{0}$ show that there exists a positive and continuous function $K_{1}$ vanishing at 0 such that

$$
\left[\left[\widetilde{g}\left(q_{2}, \mathbf{w}_{2}\right)-\widetilde{g}\left(q_{1}, \mathbf{w}_{1}\right)\right]\right]_{2} \leqslant K_{1}(\rho)\left\|\left(q_{2}, \mathbf{w}_{2}\right)-\left(q_{1}, \mathbf{w}_{1}\right)\right\|_{\mathcal{X}_{q, \mathbf{w}}},
$$

for any $\left(q_{j}, \mathbf{w}_{j}\right) \in B(\mathbf{0}, \rho)(j=1,2)$.

We now consider the operator $\widetilde{\mathcal{H}}$ (see $\sqrt{4.12}$ ). All the terms in the definition of $\widetilde{\mathcal{H}}$ belong to $C\left([0,+\infty) ; C_{b}^{\alpha}(\mathbb{R})\right) \cap C^{\alpha / 2}\left([0,+\infty) ; C_{b}(\mathbb{R})\right)$ for any $(q, \mathbf{w}) \in B(\mathbf{0}, \rho)$ for a sufficiently small $\rho$, since they are products of functions belonging to such spaces. Moreover,

$$
\begin{aligned}
& \sup _{t \geqslant 0}(1+t)^{3 / 2}\left\|\tilde{\mathcal{H}}\left(q_{2}(t, \cdot), \mathbf{w}_{2}(t, \cdot)\right)-\tilde{\mathcal{H}}\left(q_{1}(t, \cdot), \mathbf{w}_{1}(t, \cdot)\right)\right\|_{C_{b}^{\alpha}(\mathbb{R})} \\
& \quad+\sup _{s \geqslant 0}(1+s)^{3 / 2}\left[\tilde{\mathcal{H}}\left(q_{2}, \mathbf{w}_{2}\right)-\tilde{\mathcal{H}}\left(q_{1}, \mathbf{w}_{1}\right)\right]_{C^{\alpha / 2}\left([s,+\infty) ; C_{b}(\mathbb{R})\right)} \leqslant K_{2}(\rho)\left\|\left(q_{2}, \mathbf{w}_{2}\right)-\left(q_{1}, \mathbf{w}_{1}\right)\right\|_{\mathcal{X}_{q, \mathbf{w}}},
\end{aligned}
$$


for any $\left(q_{j}, \mathbf{w}_{j}\right) \in B(\mathbf{0}, \rho)(j=1,2)$ and some positive and continuous function $K_{2}$ vanishing at 0 . In particular, we get

$$
\left[\left[\tilde{\mathcal{H}}\left(q_{2}, \mathbf{w}_{2}\right)-\tilde{\mathcal{H}}\left(q_{1}, \mathbf{w}_{1}\right)\right]\right]_{0} \leqslant K_{2}(\rho)\left\|\left(q_{2}, \mathbf{w}_{2}\right)-\left(q_{1}, \mathbf{w}_{1}\right)\right\|_{\mathcal{X}_{q, \mathbf{w}}}
$$

(see 4.18). Similarly, for any $(q, \mathbf{w}) \in B(0, \rho)$ the function $\mathcal{K}(q, \mathbf{w})$ defined by (4.13)-4.15) belongs to $C^{\alpha / 2}\left([0,+\infty) ; C_{b}(\mathbb{R})\right) \cap C_{b}\left([0,+\infty) ; C_{b}^{\alpha}(\mathbb{R})\right)$. Moreover,

$$
\left[\left[\mathcal{K}\left(q_{2}, \mathbf{w}_{2}\right)-\mathcal{K}\left(q_{1}, \mathbf{w}_{1}\right)\right]\right]_{1} \leqslant K_{3}(\rho)\left\|\left(q_{2}, \mathbf{w}_{2}\right)-\left(q_{1}, \mathbf{w}_{1}\right)\right\|_{\mathcal{X}_{q, \mathbf{w}}},
$$

for any $\left(q_{j}, \mathbf{w}_{j}\right) \in B(\mathbf{0}, \rho)(j=1,2)$ (see 4.33$)$ and some positive and continuous function $K_{3}$ going to 0 as $\rho$ tends to 0 .

Now observe that, since $\widetilde{g}(0, \mathbf{0})=0, \tilde{\mathcal{H}}(0, \mathbf{0})=\widetilde{\mathcal{K}}(0, \mathbf{0})=\mathbf{0}$, from $4.71-4.73$ we deduce

$$
[[\tilde{\mathcal{H}}(q, \mathbf{w})]]_{0}+[[\tilde{\mathcal{K}}(q, \mathbf{w})]]_{1}+[[\widetilde{g}(q, \mathbf{w})]]_{2} \leqslant\left(K_{1}(\rho)+K_{2}(\rho)+K_{3}(\rho)\right)\|(q, \mathbf{w})\|_{\mathcal{X}_{q, \mathbf{w}}},
$$

for any $(q, \mathbf{w}) \in B(\mathbf{0}, \rho)$.

Now, taking Theorems 4.4, 4.7 and all the above estimates into account, we easily deduce that, if $\rho$ is sufficiently small, then $\Gamma(q, \mathbf{w}) \in \mathcal{X}_{q, \mathbf{w}}$ for any $(q, \mathbf{w}) \in B\left(\mathbf{0},\left(q_{0}, \mathbf{w}_{0}\right), \rho\right)$ (observe that the compatibility conditions in Theorem 4.6 are satisfied by virtue of 4.69$)$, since $(q(0, \cdot), \mathbf{w}(0, \cdot))=$ $\left.\left(q_{0}, \mathbf{w}_{0}\right)\right)$.

Moreover, from 4.19), 4.36, 4.74) we immediately deduce that

$$
\|\Gamma(q, \mathbf{w})\|_{\mathcal{X}_{q, \mathbf{w}}} \leqslant C\left(\left\|q_{0}\right\|_{C_{b}^{2+\alpha}(\mathbb{R})}+\left\|\mathbf{w}_{0}\right\|_{X_{2+\alpha}}+K_{4}(\rho)\|(q, \mathbf{w})\|_{\mathcal{X}_{q, \mathbf{w}}}\right),
$$

for some constant $C$, independent of $\left(q_{0}, \mathbf{w}_{0}\right)$, and some positive and continuous function $K_{4}$ vanishing at zero.

Similarly, since for any $\left(q_{1}, \mathbf{w}_{1}\right),\left(q_{2}, \mathbf{w}_{2}\right) \in B\left(\mathbf{0},\left(q_{0}, \mathbf{w}_{0}\right), \rho\right)$ the function $\Gamma\left(q_{2}, \mathbf{w}_{2}\right)-$

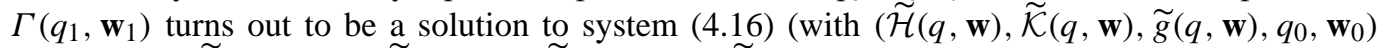
replaced by $\left.\left(\widetilde{\mathcal{H}}\left(q_{2}, \mathbf{w}_{2}\right)-\widetilde{\mathcal{H}}\left(q_{1}, \mathbf{w}_{1}\right), \widetilde{\mathcal{K}}\left(q_{2}, \mathbf{w}_{2}\right)-\widetilde{\mathcal{K}}\left(q_{1}, \mathbf{w}_{1}\right), \widetilde{g}\left(q_{2}, \mathbf{w}_{2}\right)-\widetilde{g}\left(q_{1}, \mathbf{w}_{1}\right), 0, \mathbf{0}\right)\right)$, from 4.19, 4.36, 4.71-4.73, we deduce that

$$
\left\|\Gamma\left(q_{2}, \mathbf{w}_{2}\right)-\Gamma\left(q_{1}, \mathbf{w}_{1}\right)\right\|_{\mathcal{X}_{q, \mathbf{w}}} \leqslant K_{5}(\rho)\left\|\left(q_{2}, \mathbf{w}_{2}\right)-\left(q_{1}, \mathbf{w}_{1}\right)\right\|_{\mathcal{X}_{q, \mathbf{w}}},
$$

for some positive and continuous function $K_{5}$ vanishing at zero.

Choose now a solution $\left(\rho, \rho_{0}\right)$ to the system of inequalities

$$
\left\{\begin{array}{l}
C\left(\rho_{0}+K_{4}(\rho) \rho\right) \leqslant \rho, \\
K_{5}(\rho) \leqslant 1 / 2, \\
0<\rho_{0} \leqslant \rho \leqslant 1 / 2 .
\end{array}\right.
$$

Then $\Gamma$ turns out to be a 1/2-contraction mapping in $B\left(\mathbf{0},\left(q_{0}, \mathbf{w}_{0}\right), \rho\right)$ and, consequently, the fixed point equation $\Gamma(q, \mathbf{w})=(q, \mathbf{w})$ admits a unique solution $(q, \mathbf{w}) \in B\left(\mathbf{0},\left(q_{0}, \mathbf{w}_{0}\right), \rho\right)$ solving system (4.16). Estimate (4.70) now follows easily.

\section{Concluding remarks}

The results in Section 4 imply that there exist two positive constants $\rho_{0}$ and $\rho$ such that if $\mathbf{u}_{0}$ belongs to $B\left(0, \rho_{0}\right) \subset X_{2+\alpha}$ and satisfies the compatibility conditions (4.31), then the initial value 
problem $\mathbf{u}(0, \cdot)=\mathbf{u}_{0}$ for problem 2.15 admits a unique globally defined solution $\mathbf{u} \in B(0, \rho) \subset$ $\mathcal{X}_{1+\alpha / 2,2+\alpha}(0, \infty)$. Moreover, there exists a positive constant $C$ such that

$$
(1+t)^{\alpha_{1}+\alpha_{3} / 2}\left(\left\|D_{t}^{\alpha_{1}} D_{x}^{\alpha_{2}} D_{y}^{\alpha_{3}} \mathbf{u}\right\|_{C_{b}^{\alpha / 2}\left([t,+\infty) ; X_{0}\right)}+\left\|D_{t}^{\alpha_{1}} D_{x}^{\alpha_{2}} D_{y}^{\alpha_{3}} \mathbf{u}(t, \cdot)\right\|_{X_{0}}\right) \leqslant C\left\|\mathbf{u}_{0}\right\|_{X_{2+\alpha}},
$$

for any $t \geqslant 0$ and any $2 \alpha_{1}+\alpha_{2}+\alpha_{3} \leqslant 2$.

One can show that, if $\rho_{0}$ is sufficiently small, then $\mathbf{u}$ is actually the unique solution in $\mathcal{X}_{1+\alpha / 2,2+\alpha}(0, \infty)$ to the initial value problem $\mathbf{u}(0, \cdot)=\mathbf{u}_{0}$ for problem 2.15 . To do this, the main step is to show that for any $t_{0}>0$ and any small $\mathbf{u}_{0} \in X_{2+\alpha}$ satisfying the appropriate compatibility conditions at $t=t_{0}$, the problem

$$
\begin{cases}D_{t} \mathbf{u}(t, \cdot)=\mathcal{L} \mathbf{u}(t, \cdot)+\mathcal{F}(\mathbf{u}(t, \cdot)), & t \in\left[t_{0}, t_{0}+\delta\right], \\ \mathcal{B} \mathbf{u}(t, \cdot)=\mathcal{G}(\mathbf{u}(t, \cdot)), & t \in\left[t_{0}, t_{0}+\delta\right], \\ \mathbf{u}\left(t_{0}, \cdot\right)=\mathbf{u}_{0}, & \end{cases}
$$

is uniquely solvable in a large ball of $\mathcal{X}_{1+\alpha / 2,2+\alpha}\left(t_{0}, t_{0}+\delta\right)$ for some small $\delta>0$ (independent of $t_{0}$ ). See [7, Theorem 4.1] for more details.

Coming back to problem (2.1)-(2.4) the previous results ensure that the planar TW is stable with respect to small and sufficiently smooth perturbations. In particular, the perturbed front $\phi$ stays bounded and close to the front $-t$ corresponding to the TW. Moreover, its derivatives decrease polynomially to zero. To be more precise, there exists a positive constant $C$ such that

$$
\left\|D_{t}^{\alpha_{1}} D_{y}^{\alpha_{2}}(\phi(t, \cdot)+t)\right\|_{C_{b}^{\alpha}(\mathbb{R})} \leqslant \frac{C}{(1+t)^{\alpha_{1}+\alpha_{2} / 2}},
$$

for any $t \geqslant 0$ and any $2 \alpha_{1}+\alpha_{2} \leqslant 2$. Moreover, the functions $\Theta^{1}$ and $S^{1}$ stay close to $\Theta^{0}$ and 0 , respectively, and

$$
\begin{aligned}
& \left\|D_{t}^{\alpha_{1}} D_{x}^{\alpha_{2}} D_{y}^{\alpha_{3}}\left(\Theta^{1}(t, \cdot)-\Theta^{0}\right)\right\|_{C_{b}^{\alpha}\left(\mathbb{R}_{-}^{2}\right)} \\
& \quad+\left\|D_{t}^{\alpha_{1}} D_{x}^{\alpha_{2}} D_{y}^{\alpha_{3}} S^{1}(t, \cdot)\right\|_{C_{b}^{\alpha}\left(\mathbb{R}_{-}^{2}\right)}+\left\|D_{t}^{\alpha_{1}} D_{x}^{\alpha_{2}} D_{y}^{\alpha_{3}} S^{1}(t, \cdot)\right\|_{C_{b}^{\alpha}\left(\mathbb{R}_{+}^{2}\right)} \leqslant \frac{C}{(1+t)^{\alpha_{1}+\alpha_{3} / 2}},
\end{aligned}
$$

for any $2 \alpha_{1}+\alpha_{2}+\alpha_{3} \leqslant 2$, where $\mathbb{R}_{+}^{2}:=\mathbb{R}_{+} \times \mathbb{R}$.

\section{Appendix A. Additional tools}

We begin this subsection with an abstract lemma which plays a crucial role in the proof of Theorem 4.1 .

Lemma A.1 Let $X$ be a Banach space, and $A: D(A) \subset X \rightarrow X, B: D(B) \subset X \rightarrow X$ be two closed operators with nonempty resolvent sets. Then $A$ and $B$ commute in the resolvent sense if and only if

$$
R(\omega, B)(D(A)) \subset D(A), \quad A R(\omega, B) x=R(\omega, B) A x, \quad \forall \omega \in \rho(B), \quad \forall x \in D(A) .
$$

Proof. Let us assume that $A$ and $B$ commute in the resolvent sense and prove that condition A.1 is satisfied. Fix $x \in D(A), \sigma \in \rho(A)$ and let $y \in X$ be such that $x=R(\sigma, A) y$. Then, for any $\omega \in \rho(B)$, 


$$
\begin{aligned}
R(\omega, B) A x & =R(\omega, B) A R(\sigma, A) y=R(\omega, B)(A-\sigma I+\sigma I) R(\sigma, A) y \\
& =-R(\omega, B) y+\sigma R(\omega, B) R(\sigma, A) y=-R(\omega, B) y+\sigma R(\sigma, A) R(\omega, B) y \\
& =(\sigma R(\sigma, A)-I) R(\omega, B) y=A R(\sigma, A) R(\omega, B) y=A R(\omega, B) R(\sigma, A) y \\
& =A R(\omega, B) x,
\end{aligned}
$$

so that A.1 is satisfied.

Conversely, let us prove that, if A.1 holds, then $A$ and $B$ commute in the resolvent sense. Fix $y \in X$ and take $\sigma \in \rho(A), \omega \in \rho(B)$. Applying A.1 to $x=R(\sigma, A) y$ we get

$$
\begin{aligned}
A R(\omega, B) R(\sigma, A) y & =R(\omega, B) A R(\sigma, A) y=R(\omega, B)(A-\sigma I+\sigma I) R(\sigma, A) y \\
& =-R(\omega, B) y+\sigma R(\omega, B) R(\sigma, A) y .
\end{aligned}
$$

Hence, $(A-\sigma I) R(\omega, B) R(\sigma, A) y=-R(\omega, B) y$. Applying $R(\sigma, A)$ to both sides of the previous equation we get $R(\omega, B) R(\sigma, A) y=R(\sigma, A) R(\omega, B) y$ so that $A$ and $B$ commute in the resolvent sense.

The technique we used, in the case $\lambda=0$, to transform problem 1.1 into an equivalent one, which is somewhat simpler to study, works as well in the case of problem (1.1) with $\lambda \neq 0$. We still get a problem similar to 2.15 , where now the operators $\mathcal{L}$ and $\mathcal{B}$ have to be replaced by the operators

$$
\begin{aligned}
\mathcal{L} \mathbf{u} & =\left(\Delta v-v_{x}, \Delta w-w_{x}-\lambda \Delta v, \Delta h+h_{x}\right), \\
\lambda v(0, \cdot)-w(0, \cdot)+h(0, \cdot) & \mathcal{B} \mathbf{u}=\left(\begin{array}{c}
\lambda v(0, \cdot)+\lambda v_{x}(0, \cdot)-w_{x}(0, \cdot)-h_{x}(0, \cdot) \\
v(0, \cdot)+h(0, \cdot)-v_{x}(0, \cdot)
\end{array}\right),
\end{aligned}
$$

where, as usual, we set $\mathbf{u}=(v, w, h)$. The pair $(\mathcal{L}, \mathcal{B})$ generates an analytic semigroup in $X_{0}$ for any $\lambda \in \mathbb{R}$ with domain still given by (4.1) (see [7, Theorem A.2]). We still can decouple $\mathcal{L}$ into the sum of the two operators $L_{1}$ and $L_{2}$ defined by

$$
L_{1} \mathbf{u}=\left(v_{x x}-v_{x}, w_{x x}-w_{x}-\lambda v_{x x}, h_{x x}+h_{x}\right), \quad L_{2} \mathbf{u}=\left(v_{y y}, w_{y y}-\lambda v_{y y}, h_{y y}\right),
$$

with domains

$$
D\left(L_{1}\right)=\left\{\mathbf{u}: D_{x}^{j} \mathbf{u} \in X_{0} \text { for } j=0,1,2, \mathcal{B} \mathbf{u}=0\right\}, \quad D\left(L_{2}\right)=\left\{\mathbf{u}: D_{y}^{j} \mathbf{u} \in X_{0} \text { for } j=0,1,2\right\} .
$$

Although these operators are generators of analytic semigroups in $X_{0}$, they do not commute in the resolvent sense due to the coupling between $v$ and $w$ induced by the parameter $\lambda$.

Theorem A.2 Suppose that $\lambda \neq 0$. Then the operators $L_{1}: D\left(L_{1}\right) \subset X_{0} \rightarrow X_{0}$ and $L_{2}$ : $D\left(L_{2}\right) \subset X_{0} \rightarrow X_{0}$ are generators of analytic semigroups in $X_{0}$ not commuting in the resolvent sense.

Proof. Showing that $L_{j}: D\left(L_{j}\right) \subset X_{0} \rightarrow X_{0}(j=1,2)$ generates an analytic semigroup in $X_{0}$ is an easy exercise and, hence, it is left to the reader. In particular, straightforward computations show 
that for any $\omega \in \rho(L)$ and any $\lambda \in \mathbb{R}$,

$$
\begin{aligned}
{\left[R\left(\omega, L_{1}\right) \mathbf{f}\right]_{1}(x, y)=} & \frac{1}{\sqrt{1+4 \omega}}\left(\int_{-\infty}^{0} e^{-\mu_{1} t} f(t+x, y) \mathrm{d} t+\int_{x}^{0} e^{\mu_{2} t} f(x-t, y) \mathrm{d} t\right) \\
& +a_{1} e^{x} \int_{-\infty}^{0} e^{-\mu_{1}(t+x)} g(t, y) \mathrm{d} t+a_{1} \int_{-\infty}^{0} e^{\mu_{2}(t+x)} k(t, y) \mathrm{d} t \\
& +a_{2} e^{x} \int_{-\infty}^{0} t e^{-\mu_{1}(t+x)} f(t, y) \mathrm{d} t+a_{3} e^{x} \int_{-\infty}^{0} e^{-\mu_{1}(t+x)} f(t, y) \mathrm{d} t, \\
{\left[R\left(\omega, L_{1}\right) \mathbf{f}\right]_{2}(x, y)=} & \frac{1}{\sqrt{1+4 \omega}} \int_{-\infty}^{0} e^{-\mu_{1} t}\left(g-\lambda D_{x x}\left[R\left(\omega, L_{1}\right) \mathbf{f}\right]_{1}\right)(t+x, y) \mathrm{d} t \\
& +\frac{1}{\sqrt{1+4 \omega}} \int_{x}^{0} e^{\mu_{2} t}\left(g-\lambda D_{x x}\left[R\left(\omega, L_{1}\right) \mathbf{f}\right]_{1}\right)(x-t, y) \mathrm{d} t \\
& +a_{4} e^{x} \int_{-\infty}^{0} e^{-\mu_{1}(t+x)} g(t, y) \mathrm{d} t+a_{5} \int_{-\infty}^{0} e^{\mu_{2}(t+x)} k(t, y) \mathrm{d} t \\
& +a_{6} e^{x} \int_{-\infty}^{0} t e^{-\mu_{1}(t+x)} f(t, y) \mathrm{d} t+a_{7} e^{x} \int_{-\infty}^{0} e^{-\mu_{1}(t+x)} f(t, y) \mathrm{d} t, \\
{\left[R\left(\omega, L_{1}\right) \mathbf{f}\right]_{3}(x, y)=} & \frac{1}{\sqrt{1+4 \omega}}\left(\int_{x}^{0} e^{-\mu_{1} t} k(x-t, y) \mathrm{d} t+\int_{-\infty}^{0} e^{\mu_{2} t} k(t+x, y) \mathrm{d} t\right) \\
& +a_{8} \int_{-\infty}^{0} e^{-\mu_{1}(t+x)} f(t, y) \mathrm{d} t+a_{9} \int_{-\infty}^{0} t e^{-\mu_{1}(t+x)} f(t, y) \mathrm{d} t \\
& +a_{10} \int_{-\infty}^{0} e^{-\mu_{1}(t+x)} g(t, y) \mathrm{d} t+a_{11} \int_{-\infty}^{0} e^{t-\mu_{1}(t+x)} k(t, y) \mathrm{d} t
\end{aligned}
$$

for any $(x, y) \in \mathbb{R}_{-}^{2}$ and suitable coefficients $a_{j} \neq 0(j=1, \ldots, 11)$ depending on $\lambda$, where $\mu_{j}=\frac{1}{2}+(-1) \frac{1}{2} \sqrt{1+4 \omega}$ for $j=1,2$, and $\mathbf{f}=(f, g, k)$.

An immediate computation shows that

$$
\begin{aligned}
& R\left(\omega, L_{1}\right) L_{2} \mathbf{f}=R\left(\omega, L_{1}\right)\left(\mathbf{f}_{y y}-\left(0, \lambda f_{y y}, 0\right)\right), \\
& L_{2} R\left(\omega, L_{1}\right) \mathbf{f}=R\left(\omega, L_{1}\right) \mathbf{f}_{y y}-\lambda\left(0, D_{y y}\left[R\left(\omega, L_{1}\right) \mathbf{f}\right]_{1}, 0\right),
\end{aligned}
$$

for any $\mathbf{f} \in D\left(L_{2}\right)$. Hence, $R\left(\omega, L_{1}\right) L_{2} \mathbf{f}=L_{2} R\left(\omega, L_{1}\right) \mathbf{f}$ if and only if

$$
R\left(\omega, L_{1}\right)\left(0, f_{y y}, 0\right)=\left(0, D_{y y}\left[R\left(\omega, L_{1}\right) \mathbf{f}\right]_{1}, 0\right) .
$$

Take $\mathbf{f}(x, y)=\left(0, e^{x} \widetilde{g}(y), 0\right)$ for some smooth function $g$ such that $\widetilde{g}_{y y}$ does not vanish identically. Obviously $\mathbf{f} \in D\left(L_{2}\right)$. Moreover, the derivative

$$
D_{y y}\left[R\left(\omega, L_{1}\right) \mathbf{f}\right]_{1}(x, y)=a_{1} \widetilde{g}^{\prime \prime}(y) \int_{-\infty}^{0} e^{\mu_{2}(t+x)} \mathrm{d} t, \quad \forall(x, y) \in \overline{\mathbb{R}}_{-}^{2},
$$

does not vanish identically. Hence, A.2 is not satisfied and consequently, by Lemma A.1. $L_{1}$ and $L_{2}$ do not commute in the resolvent sense. 
Lemma A.3 Let $X$ be a Banach space and let $f:[0,+\infty) \rightarrow X$ be such that $f \in C^{\alpha}([0,1] ; X)$ and

$$
\sup _{1 \leqslant s<t} s \frac{\|f(t)-f(s)\|_{X}}{(t-s)^{\alpha}}<+\infty
$$

for some $\alpha \in(0,1)$. Then $f \in C^{\alpha}([0,+\infty) ; X)$ and

$$
[f]_{C^{\alpha}([0,+\infty) ; X)} \leqslant[f]_{C^{\alpha}([0,1] ; X)}+\sup _{1 \leqslant s<t} s \frac{\|f(t)-f(s)\|_{X}}{(t-s)^{\alpha}} .
$$

In particular,

$$
\sup _{0 \leqslant s<t}(1+s) \frac{\|f(t)-f(s)\|_{X}}{(t-s)^{\alpha}} \leqslant 2\left([f]_{C^{\alpha}([0,1] ; X)}+\sup _{1 \leqslant s<t} s \frac{\|f(t)-f(s)\|_{X}}{(t-s)^{\alpha}}\right) .
$$

Proof. Since $f$ satisfies $\mathrm{A} .3$, it follows that in particular,

$$
[f]_{C^{\alpha}([1,+\infty))} \leqslant \sup _{1 \leqslant s<t} s \frac{\|f(t)-f(s)\|_{X}}{(t-s)^{\alpha}} .
$$

Moreover, if $s<1<t$, then

$$
\begin{aligned}
\|f(t)-f(s)\|_{X} & \leqslant\|f(t)-f(1)\|_{X}+\|f(1)-f(s)\|_{X} \\
& \leqslant[f]_{C^{\alpha}([0,1] ; X)}(1-s)^{\alpha}+\sup _{1 \leqslant r_{1}<r_{2}} r_{1} \frac{\left\|f\left(r_{2}\right)-f\left(r_{1}\right)\right\|_{X}}{\left(r_{2}-r_{1}\right)^{\alpha}}(t-1)^{\alpha} \\
& \leqslant\left([f]_{C^{\alpha}([0,1] ; X)}+\sup _{1 \leqslant r_{1}<r_{2}} r_{1} \frac{\left\|f\left(r_{2}\right)-f\left(r_{1}\right)\right\|_{X}}{\left(r_{2}-r_{1}\right)^{\alpha}}\right)(t-s)^{\alpha}
\end{aligned}
$$

and (A.4), A.5 easily follow.

\section{REFERENCES}

1. Agmon, S., Douglis, A., \& Nirenberg, L. Estimates near the boundary for solutions to elliptic partial differential equations satisfying general boundary conditions II. Comm. Pure Appl. Math. 17 (1964), 35-92. Zbl 0123.28706 MR 28 \#5252

2. Brauner, C.-M., Hulshof, J., \& Lunardi, A. A critical case of stability in a free boundary problem. J. Evol. Equ. 1 (2001), 85-113. Zbl 0973.35036 MR 2002f:35228

3. Brauner, C.-M. \& LunARdi, A. Instabilities in a two-dimensional combustion model with free boundary. Arch. Rat. Mech. Anal. 154 (2000), 157-182. Zbl 0984.76030 MR 2001i:35293

4. Da Prato, G. \& Grisvard, P. Sommes d'opérateurs linéaires et équations différentielles opérationnelles. J. Math. Pures Appl. 54 (1975), 305-387. Zbl 0315.47009 MR 56 \#1129

5. Kapitula, T. Multidimensional stability of planar travelling waves. Trans. Amer. Math. Soc. 349 (1997), 257-269. Zbl 0866.35021 MR 97d:35104

6. Kuramoto, Y., Chemical waves and chemical turbulence. Synergetics (Garmisch-Partenkirchen, 1977), Springer (1977), 164-173. Zbl 0369.76077 MR 58 \#25670

7. LOREnZI, L. Regularity and analyticity in a two-dimensional combustion model. Adv. Differential Equations 7 (2002), 1343-1376. MR 1920685 
8. Lorenzi, L. \& LUNARDI, A. Stability in a two-dimensional free boundary combustion model. Nonlinear Anal. 53 (2003), 227-276. Zbl 01910241 MR 1959814

9. Lorenzi, L. \& LunARdi, A. Erratum to [8]. Nonlinear Anal. 53 (2003), 859-860. MR 1971033

10. Lunardi, A., Analytic Semigroups and Optimal Regularity in Parabolic Problems. Birkhäuser, Basel (1995). Zbl 0816.35001 MR 96e:47039

11. Sivashinsky, G. I. On flame propagation under conditions of stoichiometry. SIAM J. Appl. Math. 39 (1980), 67-82. Zbl 0464.76055 MR 82b:80018 